\title{
ANALYSIS OF RESONANCES IN GRAND DESIGN SPIRAL GALAXIES
}

\author{
Nelson Vera-Villamizar and Horacio Dottori \\ Instituto de Física - Universidade Federal do Rio Grande do Sul, \\ CxP 15051, CEP 91501-970, Porto Alegre, RS, Brazil \\ Ivânio Puerari \\ Instituto Nacional de Astrofísica, Optica y Electrónica, \\ Calle Luis Enrique Erro 1, 72840 Tonantzintla, Puebla, México \\ and \\ Reinaldo de Carvalho \\ Observatório Nacional, Rua General José Cristino 77, \\ CEP 20921-400, Rio de Janeiro, RJ, Brazil
}

Received _ ; accepted 


\begin{abstract}
We have searched for corotations (CR) in three Southern grand design spiral galaxies: NGC 1365, NGC 1566 and NGC 2997. We have also introduced a method of quantifying errors in the phase diagram used to detect CR.

We established the $\mathrm{m}=2$ pattern $\mathrm{CR}$ at $12.1 \mathrm{kpc}, 9.4 \mathrm{kpc}$ and $7 \mathrm{kpc}$, for NGC 1365, NGC 1566 and NGC 2997, respectively. By using published rotation curves, we could determine spiral pattern angular speeds of $25.0 \mathrm{~km} / \mathrm{sec} / \mathrm{kpc}$, $12.2 \mathrm{~km} / \mathrm{sec} / \mathrm{kpc}$ and $17.6 \mathrm{~km} / \mathrm{sec} / \mathrm{kpc}$, respectively.

A three armed component has been detected in NGC 2997, with the CR placed at $8.7 \mathrm{kpc}$ with a pattern angular speed $\Omega_{C R_{3}}=12.7 \mathrm{~km} / \mathrm{sec} / \mathrm{kpc}$.

An $m=1$ component was detected in NGC 1566. We warily locate the CR at $7.1 \mathrm{kpc}$, with a pattern angular speed $\Omega_{C R} \simeq 16.6 \mathrm{~km} / \mathrm{sec} / \mathrm{kpc}$. This pattern does not present ILR.

Ages have been determined by studying the radial density profile of the $\mathrm{m}=2$ Fourier components in $g$ (newly formed stars) and $i$ (perturbing SDW supported by the disk of old stars), aided by the global aspect of the real spiral pattern in comparison with numerical simulations. The pattern is $\sim 1200 \mathrm{Myr}$ old in NGC 1365, 800 Myr old in NGC 1566 and younger than 80 Myr in NGC 2997.
\end{abstract}

Subject headings: Galaxies: spiral, structure, kinematics and dynamics, individual (NGC 1365, NGC 1566, NGC 2997) - Methods: numerical

\title{
1. Introduction
}

Within the framework of the Density Wave Theory (Lin \& Shu 1964), the spiral arms of disk galaxies are the manifestation of traveling waves. In a first approximation, the pattern 
speed of a density wave is constant over the disk. It is widely known that galactic disks present differential rotation and in this way, in a given galaxy, there is a radial position where the density wave pattern speed coincides with the angular velocity of the stellar disk. This radial position is know as the corotation resonance (CR) radius. The density wave pattern is slower than the disk material at radii smaller than the $\mathrm{CR}$, and it is faster at radii larger than the $\mathrm{CR}$, inducing shocks at different arms side inward and outward the CR. Other resonances can be present in a given galaxy, mainly the ILR (Inner Lindblad Resonance), and the OLR (Outer Lindblad Resonance). These resonances are placed when the condition $\Omega_{p}=\Omega(R) \pm \kappa / 2$ is satisfied. Here, $\Omega_{p}$ is the pattern speed, $\Omega(R)$ is the angular velocity of the disk, $\kappa$ is the epicyclic frequency, and the signals ' + ' and ' - ' refer to the ILR and the OLR, respectively.

Schweizer (1976) and Beckman \& Cepa (1990) have previously discussed what would be the behavior of the colors across spiral arms if a shock generated by a spiral density wave (SDW) induces star formation. The main observable characteristic of this scenario are steeper azimuthal profiles and bluer color indices on the side where the shock front is located.

Puerari \& Dottori (1997) proposed a photometric method to detect CR based on the comparison of blue and infrared frames azimuthal profiles Fourier transform, under the basic idea that a shock-induced star formation in a SDW scenario produces an azimuthal gradient of ages across the spiral arms that has opposite signs on either side of the CR.

The behavior of the azimuthal phase $\Theta(r)$ was schematically exemplified in Fig. 2 of Puerari \& Dottori (1997), for leading and trailing waves of S- and Z-type. Elmegreen, Elmegreen \& Montenegro (1992) pointed out that evidences for CR are clearly seen in gas rich galaxies in the form of sharp end points to star formation ridges and dust lanes in two armed spirals. Photometric methods are widely used to detect resonances, mainly 
due to the spar in observing time. Canzian (1998) rise some doubts on the ability of photometric methods to uniquely determine CR in M 100. For this galaxy, Elmegreen et al. (1989), using computing enhanced imagery, placed the CR radius at 110 arcsec. This value was confirmed by Sempere et al. (1995), through the HI velocity field. On the other hand, two different methods based on $\mathrm{H} \alpha$ velocity field place the CR of M 100 at around 75 arcsec (Arsenault et al. 1988; Canzian \& Allen 1997). That shows for M 100 that even the kinematical determination of CR from the velocity field of two different components do not lead to a unique result. Perhaps, the HI streaming motions over M 100 arms (Knapen et al. 1992) contribute to disturb SDW kinematics, indicating that perturbations in disks of galaxies sometimes produce more complex spatial structures and/or kinematical patterns than those suggested by simple models.

In this paper, we use and discuss again the azimuthal profiles phase method (Puerari \& Dottori 1997) to analyze the grand design spiral galaxies NGC 1365, NGC 1566 and NGC 2997, recalling the curious result on multiple CR obtained by these authors for NGC 1832 and NGC 7479. NGC 1365 and NGC 1566 belong to Elmegreen \& Elmegreen (1987) arm class 12 ('two long symmetric arms dominating the optical disk'), while NGC 2997 belongs to the arm class 9 ('two symmetric inner arms; multiple long and continuous outer arms').

Furthermore, in this paper we present a method to quantitatively determine errors. This procedure allows to determine a range of validity in radius for the azimuthal profiles phase method, a critical issue in Puerari \& Dottori (1997) Letter. We apply the azimuthal profiles phase method on the rough $g$ and $i$ images, as done by Puerari \& Dottori (1997), but also to images computer-treated with the method of Elmegreen, Elmegreen \& Montenegro (1992), and that based on modes search by two-dimensional Fourier analysis (Kalnajs 1975; Considère \& Athanassoula 1982; Puerari \& Dottori 1992). 


\section{Data and Analysis}

\subsection{Observations}

The galaxies discussed in this paper were observed in November, 1998, at the CTIO $0.9 \mathrm{~m}$ telescope. For each galaxy, three images with each of Gunn's $g$ and $i$ filters were taken, with exposure time 500 seconds each image. The standard reduction was carried out using the IRAF package ${ }^{1}$. Since our focus is morphology, no calibration were required. As a first step, all images were centered using field stars. Thereafter, field stars were removed using the IRAF imedit task. In order to enhance the perturbation over the galaxy's disks we subtract the mean radial light profile and then we normalize the rms variation of the intensity at each radius to a constant value throughout the image, as done by Elmegreen, Elmegreen \& Montenegro (1992). The galaxies were then deprojected running a program that maps ellipses into circles.

\subsection{The Azimuthal Profile Phase Method}

Shock induced star formation in a stellar density wave scenario produces an azimuthal spread of ages across the spiral arms. At the CR, the angular velocity of the perturbation $\left(\Omega_{p}\right)$ and that of the stellar disk $(\Omega)$ coincide. A comoving observer at the CR will see outward and inward the shock front to change from one side of the spiral to the other, consequently reversing the order in which young and older disk stellar population appear

\footnotetext{
${ }^{1}$ The IRAF package is written and supported by the IRAF programming group at the National Optical Astronomy Observatories (NOAO) in Tucson, Arizona. NOAO is operated by the Association of Universities for Research in Astronomy (AURA), Inc. under cooperative agreement with the National Science Foundation (NSF).
} 
in azimuthal profiles across the arms. In order to detect the shock front jump, Puerari \& Dottori (1997) proposed to analyze the relative behavior of the SDW and shock front phases respectively, by means of the Fourier transform of azimuthal profiles $I_{r}(\theta)$ given for $\mathrm{m}=2$ by

$$
F_{2}(r)=\int_{-\pi}^{+\pi} I_{r}(\theta) e^{-2 i \pi} d \theta
$$

The phase $\Theta(r)$ can be obtained for $\mathrm{m}=2$ as

$$
\Theta_{2}(r)=\tan ^{-1} \frac{\operatorname{Re}\left[F_{2}(r)\right]}{\operatorname{Im}\left[F_{2}(r)\right]}
$$

where $R e$ and $I m$ mean the real and imaginary parts of the complex Fourier coefficients. In Figs. 1 and 2 of Puerari \& Dottori (1997), one can find a graphic representation of $\Theta(r)$ behavior for 'S' and 'Z' arms of trailing and leading character. This method was also successfully used by Aguerri, Beckman \& Prieto (1998) to find CR in barred galaxies.

The SDW phase and the newly formed stars one are respectively identified with $\Theta_{d w_{2}}=\Theta_{2 i}$ and $\Theta_{s f_{2}}=\Theta_{2 g}$, where the right terms are obtained from infrared $i$ and blue $g$ images, respectively. An Azimuthal Profile Phase Difference (hereinafter, APPD) $\left[\Theta_{2 g}-\Theta_{2 i}\right]=0$, will indicate the presence of a CR.

We also search in this paper for the existence of the $\mathrm{m}=3$ component. For this component the phase $\Theta$ is given by

$$
\Theta_{3}(r)=\tan ^{-1} \frac{\operatorname{Re}\left[F_{3}(r)\right]}{\operatorname{Im}\left[F_{3}(r)\right]}
$$

where 


$$
F_{3}(r)=\int_{-\pi}^{+\pi} I_{r}(\theta) e^{-3 i \pi} d \theta
$$

\subsection{The Errors}

The basic idea for errors determination is that if the phase differences in the comparison of $g$ and $i$ images reflect an statistical fluctuation, it should also be reflected when different single filter images are compared among them. In order to quantitatively evaluate the errors intrinsic to the APPD method, we obtained three different images in $g$ and $i$ filters. We co-added $g 1+g 2$ and $g 2+g 3$ and compute the phase difference of the resulting images. The same procedure is applied to the $i$ images. When comparing single filter images of a given galaxy, the global aspect of the APPD is that of a high frequency statistical fluctuation (white noise), while APPD of $g$ against $i$ images produces low frequency phase differences, as we will see further on. We assume that an APPD that locally reach values larger than $3 \sigma$ constitutes a real signal.

\subsection{Separation of $\mathrm{m}=2$ and $\mathrm{m}=3$ Components}

In order to separate $m=2$ and $m=3$ spiral pattern components we apply two methods, namely, that of Elmegreen, Elmegreen \& Montenegro (1992) and the one based on two-dimensional Fourier transform (Puerari \& Dottori 1992).

\subsubsection{Elmegreen, Elmegreen \&3 Montenegro Method (EEM92)}

These authors propose to separate the two- and three-fold symmetric part of a galaxy spiral pattern, $\mathrm{S}_{2}$ and $\mathrm{S}_{3}$ respectively, by making successive images rotations and 
subtractions. If $I(r, \theta)$ is the original image in polar coordinates, then

$$
S_{2}(r, \theta)=I(r, \theta)-[I(r, \theta)-I(r, \theta+\pi)]_{T}
$$

and

$$
S_{3}(r, \theta)=2 I(r, \theta)-\left[I(r, \theta)+I\left(r, \theta+\frac{2 \pi}{3}\right)\right]_{T}-\left[I(r, \theta)-I\left(r, \theta-\frac{2 \pi}{3}\right)\right]_{T}
$$

where $T$ stands for truncation, meaning that pixels with negative intensities are set to 0 .

\subsubsection{The Fourier method}

This method has been extensively discussed in a number of papers (Kalnajs 1975; Considère \& Athanassoula 1982; Iye et al. 1982; Puerari \& Dottori 1992; Puerari 1993). In the Fourier method, an image is decomposed on a basis of logarithmic spiral of the form $r=r_{o} \exp \left(-\frac{m}{p} \theta\right)$. The Fourier coefficients $A(p, m)$ can be written as

$$
A(p, m)=\frac{1}{D} \int_{-\pi}^{+\pi} \int_{-\infty}^{+\infty} I(u, \theta) \exp [-i(m \theta+p u)] d u d \theta
$$

Here, $u \equiv \ln r, r$ and $\theta$ are the polar coordinates, $m$ represents the number of the arms, $p$ is related to the pitch angle $\alpha$ of the spiral as $\alpha=\operatorname{atan}(-m / p)$, and $I(u, \theta)$ is the distribution of light of a given deprojected galaxy, in a $\ln r$ versus $\theta$ plane. $D$ is a normalization factor written as

$$
D=\int_{-\pi}^{+\pi} \int_{-\infty}^{+\infty} I(u, \theta) d u d \theta
$$


Obs.: In practice, the integrals in $u \equiv \ln r$ are calculated from a minimum radius (selected to exclude the bulge where there is no information on the arms) to a maximum radius (which extends to the outer limits of the arms in our images).

The inverse Fourier transform can be written as

$$
T(u, \theta)=\sum_{m} P_{m}(u) \mathrm{e}^{i m \theta}
$$

where $P_{m}$ stands for the radial density profile of the $m$ component, written as

$$
P_{m}(u)=\frac{D}{\mathrm{e}^{2 u} 4 \pi^{2}} \int_{p_{-}}^{p_{+}} G_{m}(p) A(p, m) \mathrm{e}^{i p u} d p
$$

and $G_{m}(p)$ is a high frequency filter used to smooth the $A(p, m)$ spectra at the interval ends (Puerari \& Dottori 1992), which has the form

$$
G_{m}(p)=\exp \left[-\frac{1}{2}\left(\frac{p-p_{\max }^{m}}{25}\right)^{2}\right]
$$

where $p_{\text {max }}^{m}$ is the value of $p$ for which the amplitude of the Fourier coefficients for a given $m$ is maximum. The chosen interval ends $\left(p_{+}=50\right.$ and $\left.p_{-}=-50\right)$, as well as the step $d p=0.25$, are suitable for the analysis of galactic spiral arms.

So, by using 2D Fourier transforms, we can separate any m-mode. For example, the $\mathrm{m}=2$ mode can be analyzed using $S_{2}(u, \theta)=S_{2}(u) \mathrm{e}^{i 2 \theta}$, and so on.

\section{Spiral Structure Properties in NGC 1365}

The disk subtracted and rectified to face-on images of NGC 1365 in the $g$ and $i$ filters, are presented in Figure 1. Two dimensional Fourier analysis shows that the spiral structure 
in NGC 1365 is mainly composed of $\mathrm{m}=2$ components, in both, $g$ and $i$ frames (Figure 2). The strong signal appearing in $\mathrm{m}=4$ and $\mathrm{m}=6$ are only aliases of $\mathrm{m}=2$ (Puerari \& Dottori 1992). Nevertheless, the radial dependence of the $\mathrm{m}=2$ arm intensity $\mathrm{P}_{2}(\mathrm{r})$ is not similar in both frames, as Figure 7 shows. Up to $10 \mathrm{kpc}$, the SDW intensity, represented by the old stellar component, $\mathrm{P}_{2 i}(r)$, predominates on that of the perturbed material, represented by the newly formed stars, $\mathrm{P}_{2 g}(r)$. From $13 \mathrm{kpc}$ outwards, $\mathrm{P}_{2 i}(r)$ weakens compared to $\mathrm{P}_{2 g}(r)$. When compared with numerical models, it becomes an interesting method to determine the pattern age, as we will see in section 3.2 .

\subsection{CR Resonance in NGC 1365}

The identification of resonances in barred spirals is a matter of increasing interest for many reasons related to the SDW theory. By example, the self-consistent modeling of real systems Lindblad, Lindblad \& Athanassoula (1996), the study of the time evolution of SDW (formation, dumping, etc) and related induced star formation (Junqueira \& Combes 1996; Sellwood \& Sparke 1988; Rautiainen \& Salo 1999), and the study of non-linear coupling among different spirals modes (Tagger et al. 1987; Sygnet et al. 1988; Masset \& Tagger 1997b), are interesting, even open, problems.

Resonances in NGC 1365 have been kinematically analyzed by Jörsäter \& van Moorsel (1995) and Lindblad, Lindblad \& Athanassoula (1996). They place the CR resonance at $13.8 \mathrm{kpc}$ and $15.2 \mathrm{kpc}\left(\mathrm{R}_{C R} / \mathrm{R}_{\text {bar }} 1.15\right.$ and 1.3$)$, respectively. Lindblad, Lindblad \& Athanassoula (1996), point that in models where the disk suffers a pure bar perturbation, a pattern speed higher than $20 \pm 1 \mathrm{~km} / \mathrm{sec} / \mathrm{kpc}$ (corresponding to smaller CR radii) tends to give spirals more tighty wound than observed. They also point out that the CR radius should be even more external, if the perturbation is composed by a bar plus spiral arms. Nevertheless, as mentioned by these authors the evolution of the gaseous and/or stellar 
system to its present form is beyond the scope of their paper. This evolution precisely transforms dramatically the spiral pattern parameters (number of arms, pitch angle, etc.), distinctly in old and young components, as can be seen in Junqueira \& Combes (1996) self-consistent numerical experiments. This might explain the difference between Jörsäter \& van Moorsel (1995) and Lindblad, Lindblad \& Athanassoula (1996) results. Our results are more similar to those of Jörsäter \& van Moorsel (1995), as we will see in the next paragraph.

In Figure 3 we present the rectified $g$ image, in a $\log \mathrm{r}$ vs. $\theta$ diagram (Elmegreen, Elmegreen \& Montenegro 1992). In this plot we can see more precisely the different arm structures. The bar extends up to $10.3 \mathrm{kpc}$ and its axis is strictly straight, as can be better seen in the western arm, which is less disturbed by the dust lane. The dust lane presents an open spiral form, with pitch angle $\alpha=67^{\circ}$ with extension similar to that of the bar. The bisymmetrical arms present two different pitch angles. At the beginning it has $\alpha=16^{\circ}$, and extends itself from the end of the bar up to $17.5 \mathrm{kpc}$. The rest of the arm, up to $\approx 28 \mathrm{kpc}$, has $\alpha=40^{\circ}$. It has to be pointed out that the pitch angle change is not an artifact introduced by the galaxy warping, since the galaxy was corrected by an inclination $\omega=40^{\circ}$ and the warping does not depart more than $15^{\circ}$ from this value. Moreover, the warping begins at $\approx 25 \mathrm{kpc}$ (Jörsäter \& van Moorsel 1995).

We present in Figures 4,5 and 6 the behavior of the $\mathrm{m}=2$ APPD of the original images $\left(\left[\Theta_{2 g}-\Theta_{2 i}\right]_{\text {orig }}\right)$, and those transformed by EEM92 $\left(\left[\Theta_{2 g}-\Theta_{2 i}\right]_{e e m}\right)$ and Fourier $\left(\left[\Theta_{2 g}-\Theta_{2 i}\right]_{F o u r}\right)$ methods. The corresponding errors in $g$ and $i$, are obtained according to the prescriptions of Section 2.3.

In Figure 4, one can see that inside $5 \mathrm{kpc}$ the $\mathrm{S} / \mathrm{N}$ is too high, mainly in the $i$ band, as to advance any conclusion. Between $\approx 6 \mathrm{kpc}$ and $15 \mathrm{kpc},\left[\Theta_{2 g}-\Theta_{2 i}\right]$ comfortably overcome the noise by many $\sigma$. Beyond $15 \mathrm{kpc}$, it is again strongly affected by the noise. The CR 
at the extreme of the bar is set at $10.7 \mathrm{kpc}$ by this method. The more internal cuts are probably produced by the influence of the dust lane, whose curvature is different from that of the stellar bar.

$$
\left[\Theta_{s f}-\Theta_{d w}\right]_{e e m} \text { and }\left[\Theta_{s f}-\Theta_{d w}\right]_{F o u r} \text { present a similar behavior than that of the }
$$

original images, but with a better $\mathrm{S} / \mathrm{N}$. The $\mathrm{CR}$ is set at 13.8 and $11.8 \mathrm{kpc}$ respectively by these two methods, with a mean value in close agreement to Jörsäter \& van Moorsel (1995) determination. Both methods show a tendency to unravel a CR at $25 \mathrm{kpc}$, distance at which the disk begin to warp. But the error in the $i$ image increase strongly, and prevent any conclusion on the reality of this CR. Deeper imagery could help to better understand the SDW behavior in this region.

According to the rotation curve of Persic \& Salucci (1995), the mean value for the CR radius of $\mathrm{r}_{C R}=12.1 \mathrm{kpc}$ leads to a pattern speed $\Omega_{\text {Pattern }}=25.0 \mathrm{Km} / \mathrm{s} / \mathrm{kpc}$.

We have searched for the presence of $\mathrm{m}=3$ component, by means of EEM92 and Fourier transform methods, but this component is not significant in NGC 1365 (see the spectrum for $\mathrm{m}=3$ in Fig. 2). The $\mathrm{m}=1$ component appears to weak in the Fourier analysis, and prevent any CR study.

\subsection{NGC 1365 Spiral Pattern Age}

Junqueira \& Combes (1996) performed numerical models of disk galaxies with $\mathrm{n}=1$ Toomre's disks of stars and gas. In all the experiments they used a radial scale length, $\mathrm{a}_{s}=3.5 \mathrm{kpc}$ and $4.0 \mathrm{kpc}$, for the star disk, and $\mathrm{a}_{g}=6.0 \mathrm{kpc}$ for the gaseous disk. The extend of their stellar and gaseous disks is $12 \mathrm{kpc}$ and $16 \mathrm{kpc}$, respectively. Their models were completed with a Plummer's bulge with scale length, $\mathrm{a}_{b}$ ranging from $1 / 4.5$ to $1 / 3$ of $\mathrm{a}_{s}$, and mass equal to that of the stellar disk. These models do not have halo. Junqueira \& Combes 
(1996) present the evolution of the stars and gas spiral patterns between $20 \mathrm{Myr}$ and 2000 Myr for ten different experiments. NGC 1365 compares very well to their experiment E. It has $\mathrm{a}_{s}=3 \mathrm{kpc}$, and $\mathrm{a}_{b}=1$. In this numerical experiment, they found that the arm structure, which is multiple armed at $200 \mathrm{Myr}$, becomes two-armed at $800 \mathrm{Myr}$. From there on, the radial behavior of the $\mathrm{m}=2$ Fourier intensity of young $\mathrm{P}_{2 g}$ and old $\mathrm{P}_{2 i}$ stellar population, which were fairly similar at $200 \mathrm{Myr}$, begin to differentiate between them. $\mathrm{P}_{2 i}$ predominates in the inner $1 / 4$ of the disk length, and $\mathrm{P}_{2 g}$ outwards. We compare $\mathrm{P}_{2 g}$ and $\mathrm{P}_{2 i}$ in Figure 7, where we plotted corresponding Fourier radial intensities for perturbing and perturbed material (Junqueira \& Combes 1996), after 1200 Myr (their Figure 10). As can be seen, the qualitative behavior is quite similar to that of NGC 1365, giving to this diagram a good chance as a model dependent spiral pattern age indicator. These experiments are specially suited for NGC 1365 because this galaxy does not possess an important halo, as

clearly demonstrated by the highly Keplerian behavior of the rotation curve for $\mathrm{r}>\approx 20 \mathrm{kpc}$ (Jörsäter \& van Moorsel 1995).

\section{Spiral Structure Properties of NGC 1566}

The images in the $g$ and $i$ filters for NGC 1566, disk subtracted and rectified to face-on, are presented in Figure 8.

The rotation curve of this galaxy (Persic \& Salucci 1995) presents a high dispersion, because it is projected practically face-on on the sky . For this reason, a photometric analysis of the resonances is very appropriated in the case of NGC 1566 (Elmegreen \& Elmegreen 1990).

The HII regions spiral pattern of this galaxy was already analyzed using Fourier transform by Puerari \& Dottori (1990). The predominant pattern of this young stellar 
population is $\mathrm{m}=2$, with $\mathrm{m}=1$ being progressively more important at radii larger than $17 \mathrm{kpc}$. The present analysis of $g$ and $i$ imagery shows up the same property (Figure 9). Contrary to the case of NGC 1365, in NGC 1566 the $\mathrm{m}=2$ SDW intensity in the old stellar component $\mathrm{P}_{2 i}$ does not predominates in any part of the disk on that of the newly formed stars, $\mathrm{P}_{2 g}$ (Figure 14). Both functions decrease similarly and fade up simultaneously at about $22 \mathrm{kpc}$.

For the one armed component, we note that between 10 and $13 \mathrm{kpc} \mathrm{P}_{1 i}$ dominates on $\mathrm{P}_{1 g}$, while in more external regions the contrary happens (Figure 15).

\subsection{CR Resonance in NGC 1566}

The resonances of NGC 1566 were studied by Elmegreen \& Elmegreen (1990). The method they used was an identification of optical features together with constrains set by plausible rotation curves. They fitted radii for the ILR, OLR, CR, and other resonances.

As in the case of NGC 1365, in Figures 11, 12, 13 we present the behavior of the APPD for the three methods used in this analysis. As before, the corresponding errors in $g$ and $i$, are obtained according to the prescriptions of Section 2.3.

Each one of the methods respectively places the $\mathrm{CR}$ at $\mathrm{R} \approx 9.7 \mathrm{kpc}$ (Fig. 11), $\mathrm{R} \approx$ $8.5 \mathrm{kpc}$ (Fig. 12), and $\mathrm{R} \approx 10 \mathrm{kpc}$ (Fig. 13) in good agreement among them. This distance corresponds to the CR assumed by Elmegreen \& Elmegreen (1990). While some other intersections can be seen in the diagrams, the $\mathrm{S} / \mathrm{N}$ indicates that their evidence is marginal.

In Fig. 10 we present the rectified image in a $\log \mathrm{r}$ vs. $\theta$ diagram. As we can see, at the CR circle the main arms begin to thick outwards. The same behavior can be seen in the $\log \mathrm{r}$ vs. $\theta$ diagram of the symmetrized S2 image (not presented here). Other important property of this galaxy is that the arms, although ill defined between 10 and $15 \mathrm{kpc}$, are logarithmic 
between 5 and $22 \mathrm{kpc}$, with a pitch angle $\alpha \simeq 36^{\circ}$. A short secondary arm, parallel to the main one, appears between 12 and $22 \mathrm{kpc}$. It is better defined in the SW arm, appearing as a rather asymmetric structure. Surprisingly a similar structure appears at 800 Myr in Junqueira and Combes experiment F2 (their Fig. 19), in agreement with the age determined in the next section for the SDW in this galaxy. The arms bend themselves between 20 and $24 \mathrm{kpc}$, with a different pitch angle, although the feature is too weak to further analysis. We search also for $\mathrm{m}=3$ component in NGC 1566, but it is not important. On the other hand, an $\mathrm{m}=1$ component is present, although tenuously defined. One can warily locate its CR at $7.1 \mathrm{kpc}$, without ILR, and pattern angular speed $\Omega_{C R}=16.6 \mathrm{~km} / \mathrm{sec} / \mathrm{kpc}$.

\subsection{NGC 1566 Spiral Pattern Age}

As in the case of NGC 1365, we compared the spiral pattern properties with Junqueira \& Combes (1996) models. Odd SDW components in Junqueira \& Combes (1996) models appear at earlier evolutionary stages of the perturbation. Later on, the model spiral structure transforms itself into a two armed one. The more suitable model for the case of NGC 1566 is that of their experiment F, with $\mathrm{n}=1$ Toomre's disks of stars and gas, and radial scale length of the star and gaseous disks, $\mathrm{a}_{s}=4.0 \mathrm{kpc}$, and $\mathrm{a}_{g}=6.0 \mathrm{kpc}$, respectively. The extend of the stellar and gaseous disks is $12 \mathrm{kpc}$ and $16 \mathrm{kpc}$, respectively. The Plummer's bulge scale length for this model is $\mathrm{a}_{b}=\mathrm{a}_{s} / 4$, and the bulge mass is equal to that of the stellar disk.

The behavior of $\mathrm{P}_{2 g}$ and $\mathrm{P}_{2 i}$ (Figure 14) as well as the Fourier images of old and young stars are compatible with an SDW age of about 800 Myr in this galaxy. We do not know how important the halo is in this galaxy, in order to evaluate if the models are so suitable as in the former case. Nevertheless, Parkes $21 \mathrm{~cm}$ observations (Becker et al. 1988) indicate that the HI extends up to $90 \%$ of the optical disk, pointing also in this case to a not too 
much extended halo.

\section{Spiral Structure Properties of NGC 2997}

We present in Figure 16 the disk subtracted and deprojected to face-on image of NGC2997.

This galaxy is not as symmetric as NGC 1365 and NGC 1566. As discussed before, NGC 2997 is classified by Elmegreen \& Elmegreen (1987) as arm class 9 ('two symmetric inner arms; multiple long and continuous outer arms'), while NGC 1365 and NGC 1566 are both arm class 12 ('two long symmetric arms dominating the optical disk').

Fourier analysis of HII regions was performed by Considère \& Athanassoula (1982), Puerari \& Dottori (1992) and García-Gómez \& Athanassoula (1993). All studies show a predominance of $\mathrm{m}=2$ and $\mathrm{m}=3$ modes. At some radii, $\mathrm{m}=3$ is even stronger than $\mathrm{m}=2$. Near-infrared images of NGC 2997 (Block \& Puerari 1999), reveal a strong asymmetry on the main inner arms, suggesting the presence of an $m=1$ mode, besides the $m=3$ mode, in the older disk population (Block et al. 1994). The Fourier bisymmetric component in a $\mathrm{K}^{\prime}$ image shows a pitch angle $\alpha=25^{\circ}$.

In Figure 17 we present the Fourier spectra for the $g$ and $i$ images of NGC 2997. The $\mathrm{m}=2$ mode is, as expected, the predominant one. It is important to note that the $\mathrm{m}=1$ component, which appears at the noise level in the $g$ image, presents a reasonable weight with respect to $\mathrm{m}=3$ in $i$ image, although weaker than $\mathrm{m}=3$. Since in $\mathrm{K}^{\prime}, \mathrm{m}=1$ dominates on $\mathrm{m}=3$ (Block \& Puerari 1999), and $\mathrm{m}=3$ has practically a color independent weight relative to $\mathrm{m}=2$, it is strongly suggesting that the $\mathrm{m}=1$ component is the only one really affected by dust obscuration. 


\subsection{CR Resonances in NGC 2997}

It was very difficult to apply the Phase method to the original images of NGC 2997. The $\mathrm{m}=2$ phase did follow the internal arms, but becomes very chaotic at the distance of the arm bifurcation. The presence of several strong interarm features also contributes to increase the noise and makes the results less reliable. So, the methods that involve image processing, namely EEM92 and Fourier transform, are more suitable in the case of NGC 2997.

To treat properly the main $\mathrm{m}=2$ component, the part of the bisymmetric image in Figure 19 containing the secondary $\mathrm{m}=2$ component in the $\mathrm{E}-\mathrm{W}$ direction, from $7 \mathrm{kpc}$ outwards, was erased. Figure 20 shows the APPD for the main $\mathrm{m}=2$ component. There is a clear cut at $7 \mathrm{kpc}$, with very good $\mathrm{S} / \mathrm{N}$. The $\mathrm{CR}$ is located where the EW extension of the $\mathrm{m}=2$ arms appears (Figure 19). This can be easily seen in Figure 18, where we plotted the symmetrized image in $\log \mathrm{r}$ versus $\theta$. From the rotation curve of Sperandio et al. (1995), we derived a 2-arms SDW angular velocity of $\Omega_{S D W 2}=18 \mathrm{~km} / \mathrm{s} / \mathrm{kpc}$. The same place for the $\mathrm{m}=2 \mathrm{CR}$ position is found from Fourier analysis (Figure 21).

EEM92 method clearly reveals in this case the presence of a 3 -arms component (Figure 23), each arm covering an arch of $\approx 180^{\circ}$ between $3 \mathrm{kpc} \lesssim \mathrm{r} \lesssim 22 \mathrm{kpc}$. Similar 3-arms morphology has been detected by EEM92 in NGC 628, NGC 1232, NGC 5457 and NGC 6912. The global sense of winding of this component is the same as that of the 2-armed one. Nevertheless, in the case of NGC 2997 the 3-arms are too wide at the beginning and present in this region a remarkable concavity of leading nature between $7 \mathrm{kpc} \lesssim \mathrm{r} \lesssim 11 \mathrm{kpc}$. We dettached the concavity with a filled curve in Figure 23. The blow-up in the figure

shows details of the ridge of one of the 3 -arms concavity. APPD diagram (Figure 24), shows a intersection at $8.5 \mathrm{kpc}$, where the phase shift crosses downward, in agreement with the leading character of the described 3 -arms concavity, indicating that star formation occurs at 
the ridge crests. The detection of the elusive 3-armed SDW CR resonance in this galaxy is one important point of this study. Nevertheless, a better understanding would be necessary of what the phase diagram should do for a 3-armed component.

From the rotation curve of Sperandio et al. (1995) we obtained a pattern speed for the 3-arms SDW $\Omega_{S D W 3} \approx 13.7 \mathrm{~km} / \mathrm{s} / \mathrm{kpc}$.

In Figure 18 we present NGC 2997 rectified image of the $\mathrm{m}=2$ component as derived from EEM92 method, in a $\log \mathrm{r}$ vs $\theta$ diagram. The bifurcation of the $\mathrm{m}=2$ component begins at the $\mathrm{CR}$ radius $\left(\mathrm{r}_{c r}=7 \mathrm{kpc}\right)$. The main $\mathrm{m}=2$ spiral extends up to $\mathrm{r}=16 \mathrm{kpc}$ and the secondary between $7 \mathrm{kpc}$ and $21 \mathrm{kpc}$. Both spirals are logarithmic, with pitch angle $\alpha=15^{\circ} .7$ and $\mathrm{P}=39^{\circ} .4$, respectively.

\subsection{NGC 2997 Spiral Pattern Age}

We compare the images and $\mathrm{m}=2$ Fourier density profiles of NGC 2997 (see Figure 22) with Junqueira \& Combes (1996) models. Here, the presence of asymmetries and higher m modes betrays the young age of the perturbation in NGC 2997. As discussed above, odd SDW components in Junqueira \& Combes (1996) models appear at earlier evolutionary stages of the perturbation. In this case, it is difficult to assign an specific model from Junqueira \& Combes (1996) which fits NGC 2997. Models E, F, G, and H show similar features at stages younger than $80 \mathrm{Myr}$. The $\mathrm{m}=2$ Fourier radial density profiles present two differentiated parts: Inside $14 \mathrm{kpc} \mathrm{P}_{2 g} \approx \mathrm{P}_{2 i}$, while outwards the old population function predominates on that of the young one, probably indicating that the external parts of the arms may have been produced by an older SDW. This last assessment has to be taken with caution, since Junqueira \& Combes (1996) model F that fits this galaxy, seems to be oversimplified to explain the scenario of the SDW in NGC 2997. 


\section{Discussion and Conclusions}

The one-D Fourier transform method, applied to $g$ (blue) and $i$ (infrared) images proposed by Puerari \& Dottori (1997) to detect CR, has been applied to three grand design galaxies, namely, NGC 1365, NGC 1566 and NGC 2997. Some improvements have been introduced in this paper, to make the analysis more reliable. The original treatment on rough images of Puerari \& Dottori (1997), was this time complemented with the analysis of computer treated images, with 2-D Fourier transform and EEM92 methods. This complete procedure presents complementary aspects, which gives advantages with respect to the original one. For example, to work on the original image is very good for pure $\mathrm{m}=2$ arms. When other components appear, Fourier transform and EEM92 methods work better. Fourier method gives smaller errors than EEM92 method, but EEM92 method is more realistic than the former, since it is not constrained to describe the spiral as pure logarithmic ones. EEM92 method also have detected nicely the presence of the $\mathrm{m}=3$ component in NGC 2997. This method is extremely efficient in detecting this component, while Fourier methods has to add many components. So, we confident can say that $\mathrm{m}=3$ is not present in NGC 1365 and NGC 1566, and also that NGC 1566 presents an $\mathrm{m}=1$ component.

The method presented here to determine errors in the APPD diagram, also represents a considerable improvement with respect to the original paper (Puerari \& Dottori 1997), that strongly helps to identify which is the level of confidence in a CR determination. In turn, it also helps to establish the correct observational time to verify the dubious cases. From this point of view, it will be important to check Puerari \& Dottori (1997) result on multiple CR in the galaxies NGC 1832 and NGC 7479. Nevertheless, more images of these galaxies are

necessary to obtain a $\mathrm{S} / \mathrm{N}$ in $\mathrm{APPD}$ diagram. The present form of this diagram, as a phase difference rather than as the superposition of the phases in each color, is more instructive than the former one, specially to visualize the $\mathrm{S} / \mathrm{N}$. 
The determination of the arms extend has been made via $\log \mathrm{r}$ vs. $\theta$ diagram and via rotation curve, once know the $\mathrm{CR}$ radius.

The arm complexity as well as the relative arm intensity for perturbing SDW $\mathrm{P}_{2 i}(\mathrm{r})$

and perturbed material $\mathrm{P}_{2 g}(\mathrm{r})$ in the Fourier diagram are related to the perturbations age. Although model dependent, a comparison with suitable models, allows to establish a scale of ages for the galaxies analyzed here. That leads to think that these three objects present different phases of a single phenomenon, in spite of the strong bar in NGC 1365. These conclusions have to be further checked with more elaborated models and for a larger sample of galaxies.

\section{Acknowledgements}

Nelson Vera-Villamizar thanks a studentship of the Brazilian Foundation CNPq. This research is partially supported by the Mexican Foundation CONACYT under the grant No. 28507-E and by the Brazilian institutions CNPq and CAPES under the program PRONEX grant No. 76.97.1003.00. 


\section{REFERENCES}

Aguerri, J.A.L., Beckman, J.E., Prieto, M., 1998, AJ 116, 2136.

Arsenault, R., Boulesteix, J., Georgelin, Y., Roy, J.-R., 1988, A\&A 200, 29.

Becker, R., Meblod, U., Reif, K. van Woerden, H., 1988, A\&A 203, 21.

Beckman, J.E., Cepa, J. 1990, A\&A 229, 37.

Block, D.L., Puerari, I., 1999, A\&A 342, 627.

Block, D.L., Bertin, G., Stockton, A., Grosbøl, P., Moorwood, A.F.M., Peletier, R.F., 1994, A\&A 288, 383.

Canzian, B. 1998, ApJ 502, 582.

Canzian, B., Allen, R.J. 1997, ApJ 479, 723.

Considère, S., Athanassoula, E. 1982, A\&A 111, 28.

Elmegreen, B.G., Elmegreen, D.M. 1990, ApJ 355, 52.

Elmegreen, B.G., Elmegreen, D.M., Seiden, P.E., 1989, ApJ 343, 602.

Elmegreen, B.G., Elmegreen, D.M., Montenegro, L., 1992, ApJS 79, 37 (EEM92).

Elmegreen, D.M., Elmegreen, B.G., 1987, ApJ 314, 3.

Iye, M., Okamura, S., Hamabe, M., Watanabe, M., 1982, ApJ 256, 103.

García-Gómez, C., Athanassoula, E., 1993, A\&AS 100, 431.

Jörsäter, S., van Moorsel, G.A., 1995, AJ 110, 2037.

Junqueira, S., Combes, F., 1996, A\&A 312, 703. 
Kalnajs A.J., 1975, in La Dynamique des Galaxies Spirales, ed. L. Weliachew (Paris Editions du CNRS), 103.

Knapen, J.H., Beckman, J.E., Cepa, J., van der Hulst, T., Rand, R.J., 1992, ApJ 385, L37.

Knapen, J.H., Beckman, J.E., Cepa, J., Nakai, N., 1996, A\&A 308, 27.

Lin, C.C., Shu, F.H., 1964, ApJ 140, 646.

Lindblad, P.A.B., Lindblad, P.O., Athanassoula, E., 1996, A\&A 313, 65.

Masset, F., Tagger, M. 1997a, A\&A 318, 747.

Masset, F., Tagger, M. 1997b, A\&A 322, 442.

Patsis, P., Grosbøl, P., Hiotelis, N., 1997, A\&A 323, 762.

Persic, M., Salucci, P., 1995, ApJS 99, 501.

Puerari, I., 1993, PASP 105, 1290.

Puerari, I., Dottori, H., 1990, RMxAA 21, 126.

Puerari, I., Dottori, H.A., 1992, A\&AS 93, 469.

Puerari, I., Dottori, H., 1997, ApJ 476, L73.

Rautiainen, P., Salo, H., 1999, A\&A 348, 737.

Schweizer, F., 1976, ApJS 31, 313.

Sellwood, J.A., Sparke, L.S., 1988 MNRAS 231, 25p.

Sempere, M. J., Combes, F.,Casoli, F., 1995, A\&A 299, 37.

Sperandio, M., Chincarini, G., Rampazo, R., De Souza, R., 1995, A\&AS 110, 279. 
Sygnet, J.F., Tagger, M., Athannasoula, E., Pellat, F., 1988, MNRAS 232, 733.

Tagger, M., Sygnet, J.F., Athanassoula, E., Pellat, F., 1987, ApJ 318, L43. 
(a)

(b)

Fig. 1.- NGC 1365 disk subtracted and rectified to face-on images according to procedure described in section 2.1. (a) $g$ image, (b) $i$ image. 

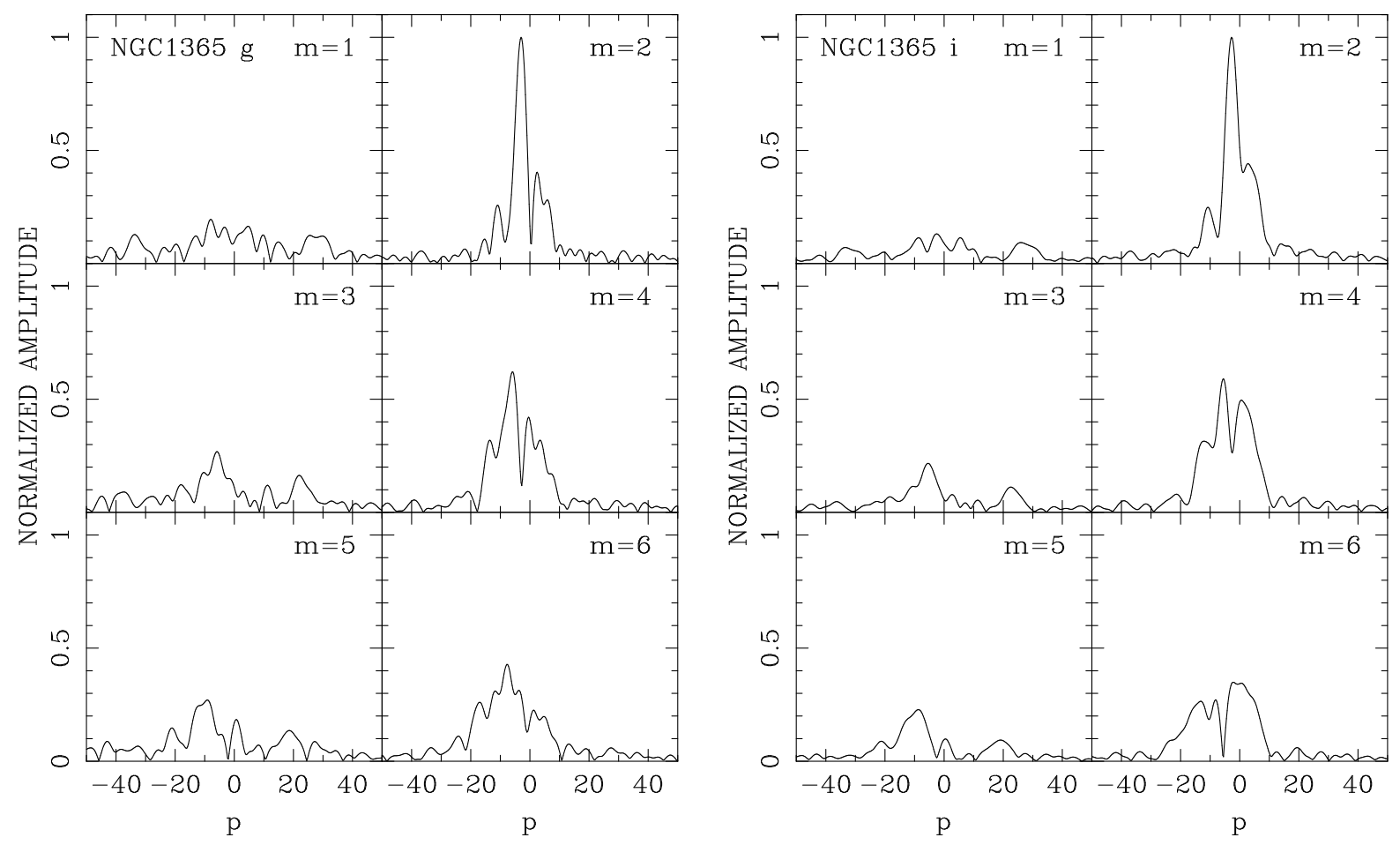

Fig. 2.- Two-dimensional Fourier spectra coefficients $A(p, m)$ of NGC 1365, obtained as described in section 2.4.2. Left, $g$ image, and right $i$ image. The $p$ variable is related to the pitch angle $\alpha$ by $p=-\frac{m}{\tan \alpha}$. 
Fig. 3. - NGC $1365 g$ band rectified image in $\log \mathrm{r}$ versus $\theta$ diagram. The horizontal line shows the CR loci at 13 kpc. According to Elmegreen, Elmegreen \& Montenegro (1992)(Hereinafter EEM92). 


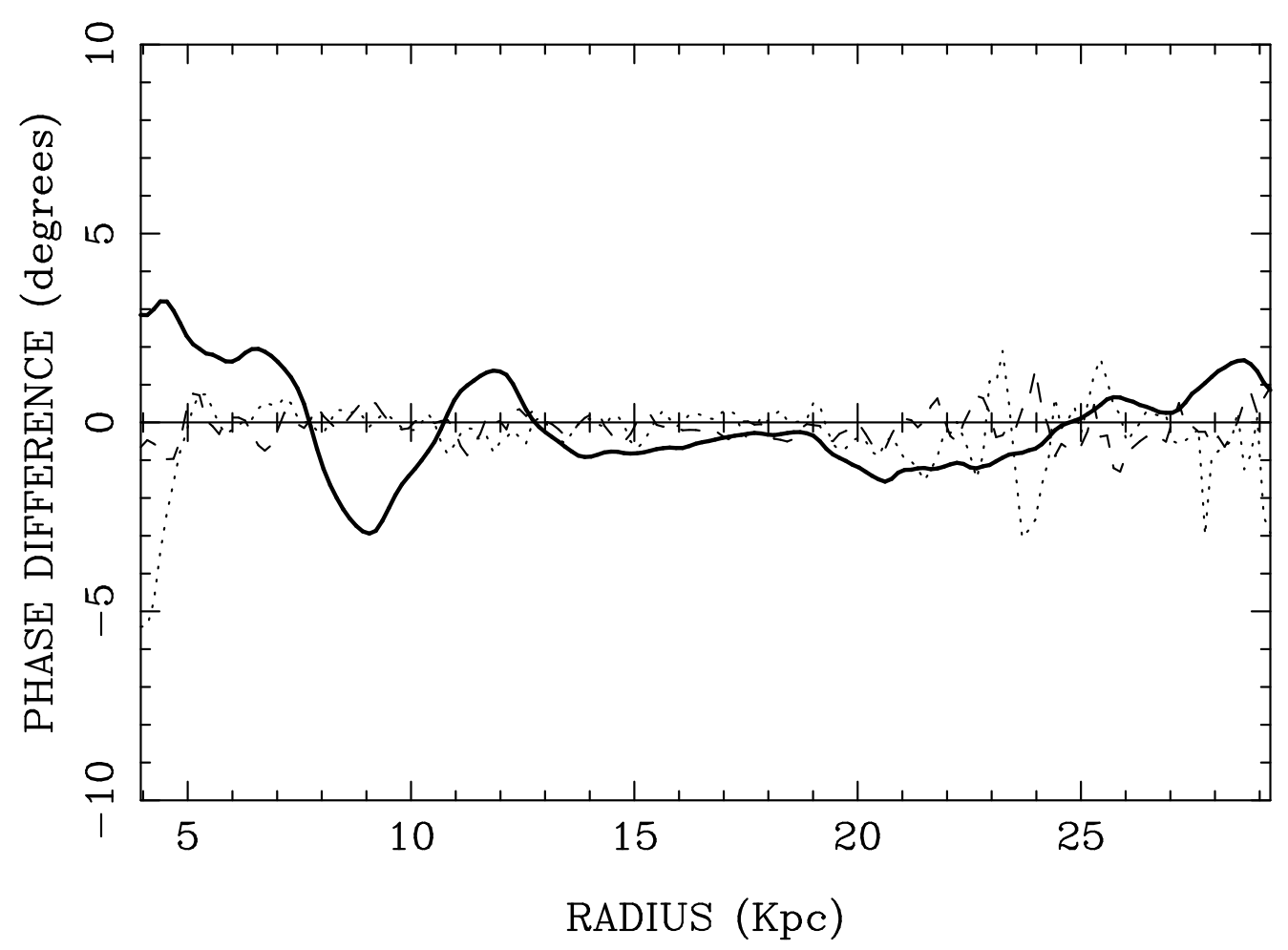

Fig. 4.- Azimuthal Profile Phase Difference (APPD) $\left[\Theta_{2 g}-\Theta_{2 i}\right]$ for NGC 1365 obtained from original images (solid line). Broken line shows $g$ noise and dotted line shows $i$ noise. 


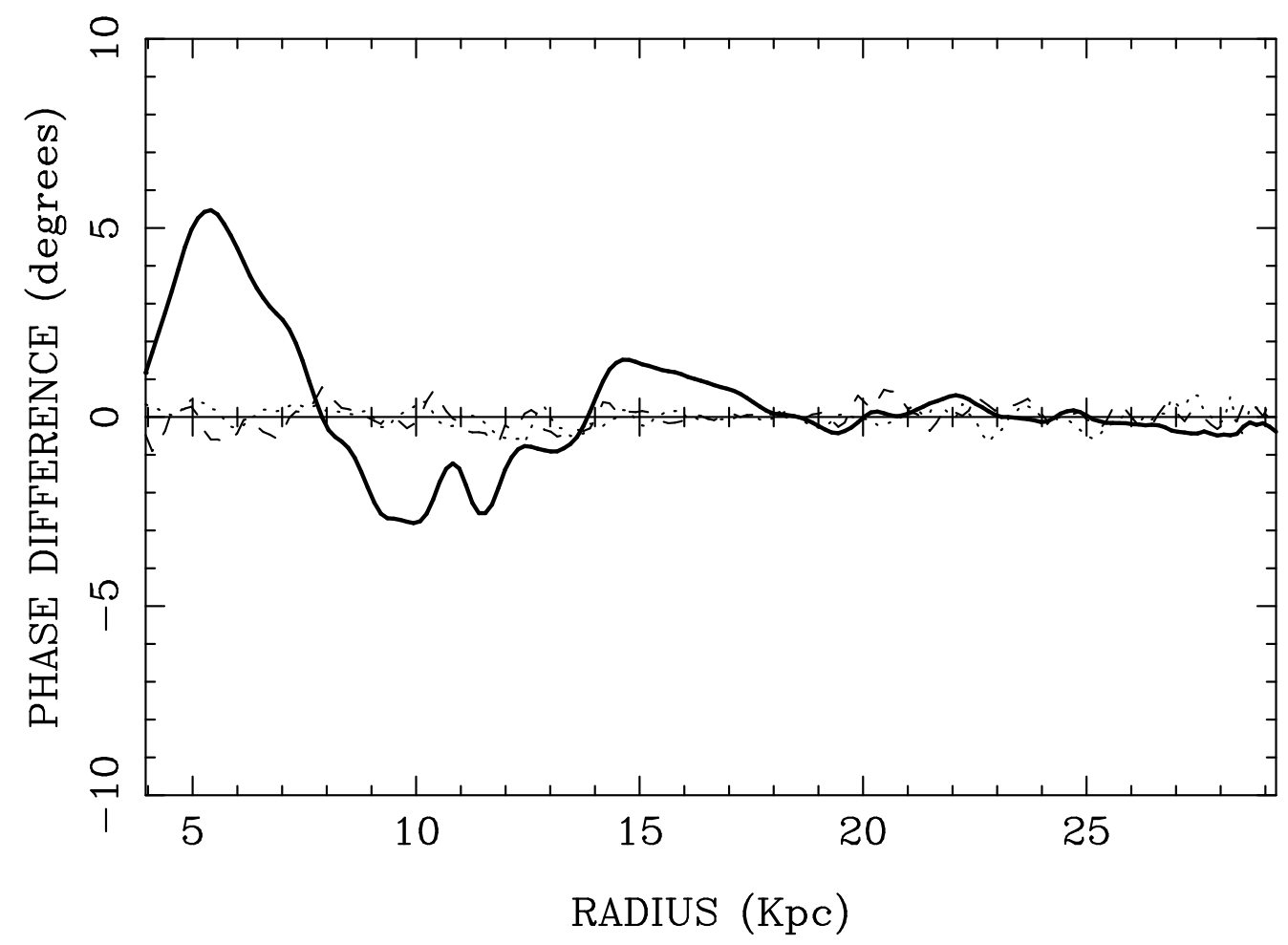

Fig. 5.- APPD $\left[\Theta_{2 g}-\Theta_{2 i}\right]$ for NGC 1365 obtained from images symmetrized according to EEM92 Method (solid line). Broken line shows the noise in $g$ color and dotted line shows that in $i$ color. See sections 2.4 .1 and 2.3. 


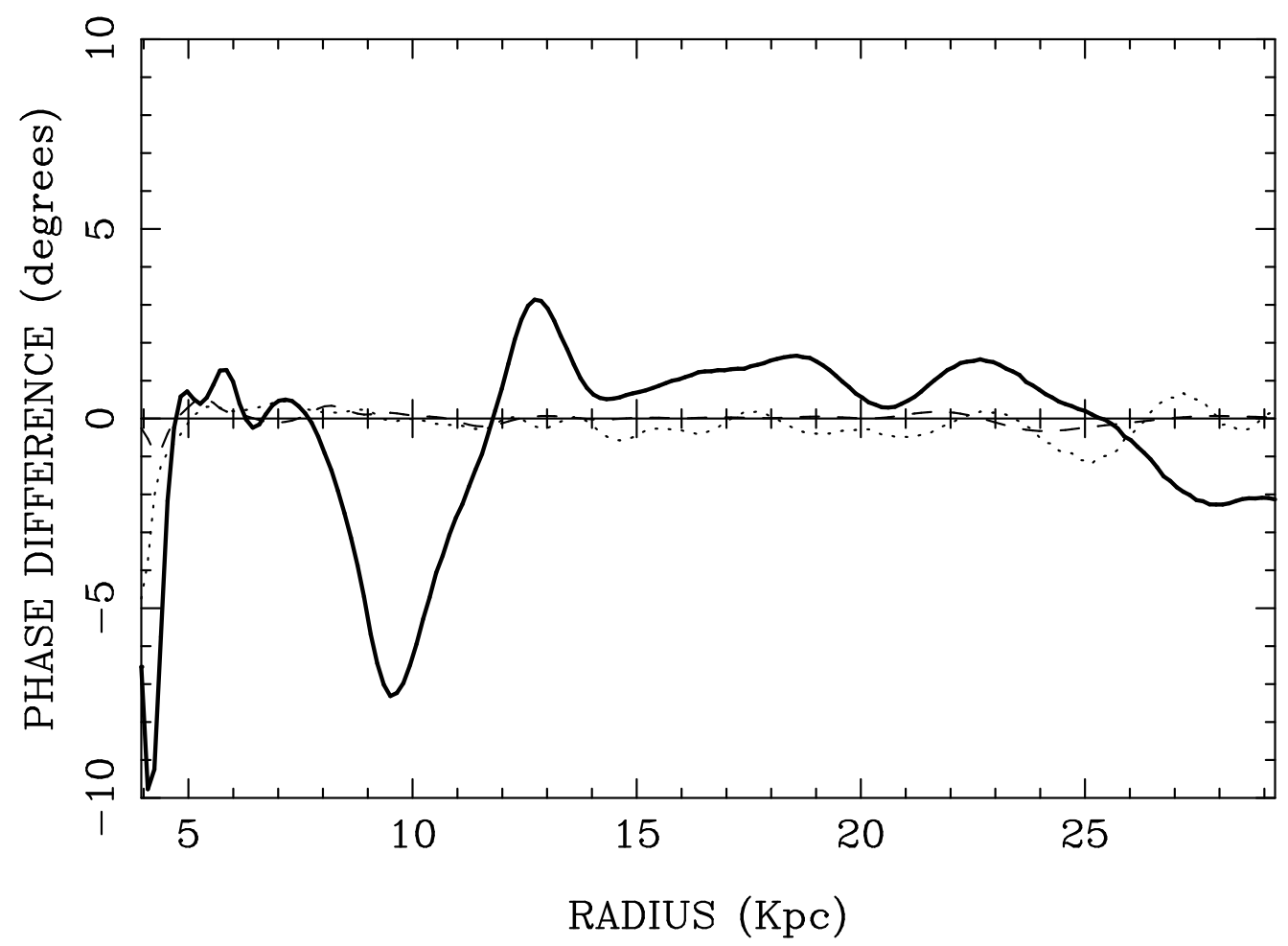

Fig. 6. - APPD $\left[\Theta_{2 g}-\Theta_{2 i}\right]$ for NGC 1365 obtained from the Fourier $\mathrm{m}=2$ modes (solid line). Broken line shows $g$ noise and dotted line shows $i$ noise. See sections 2.4.2 and 2.3. 


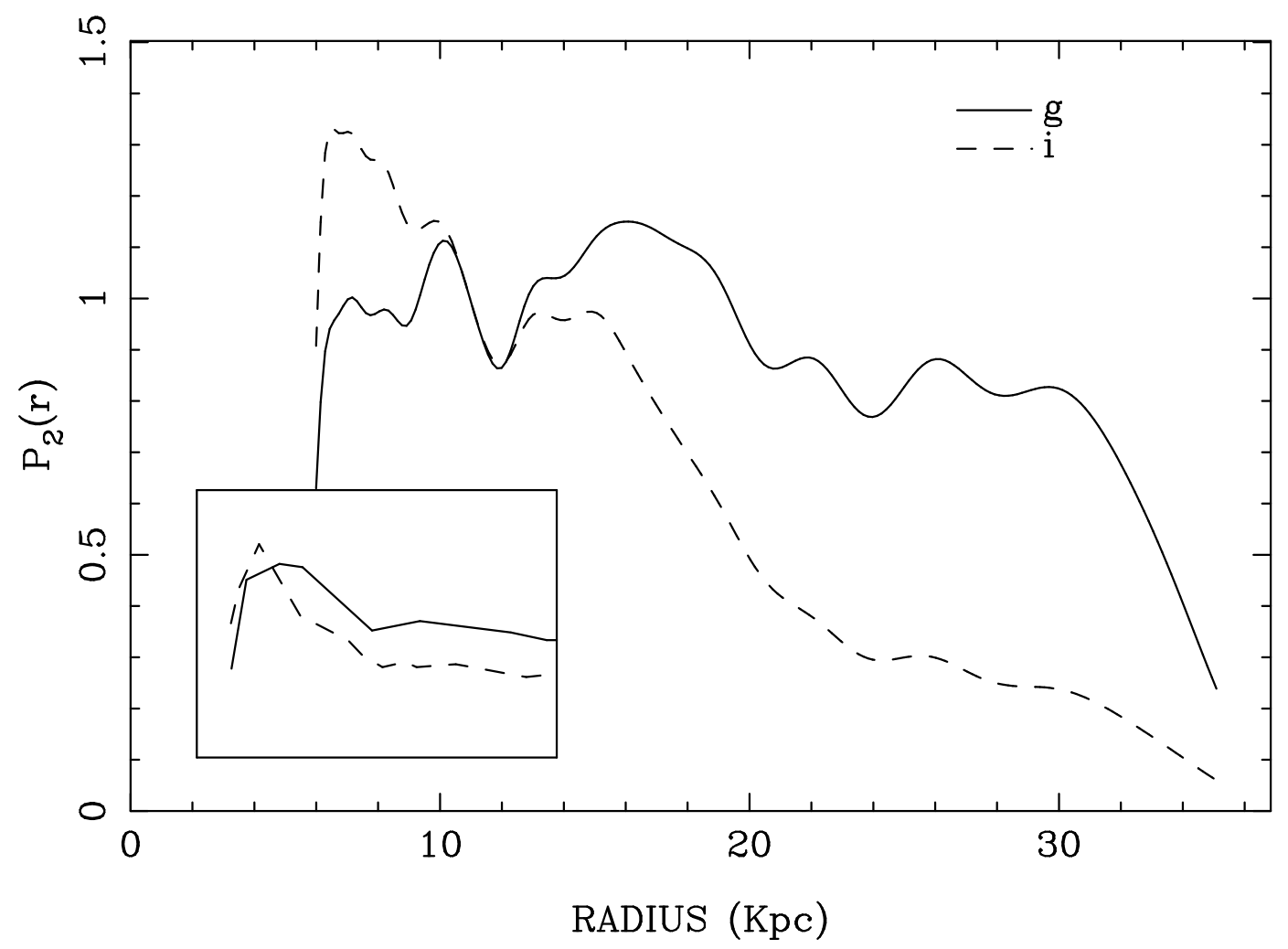

Fig. 7.- Comparison of Fourier radial density functions in blue $P_{2 g}$ and infrared $P_{2 i}$ (see section 2.4.2) for NGC 1365. Inset shows SDW and gas Fourier radial density functions for Junqueira \& Combes (1996) model E after 1200 Myr. 
(a)

(b)

Fig. 8.- NGC 1566 disk subtracted and rectified to face-on images according to procedure described in section 2.1. (a) $g$ image, (b) $i$ image. 

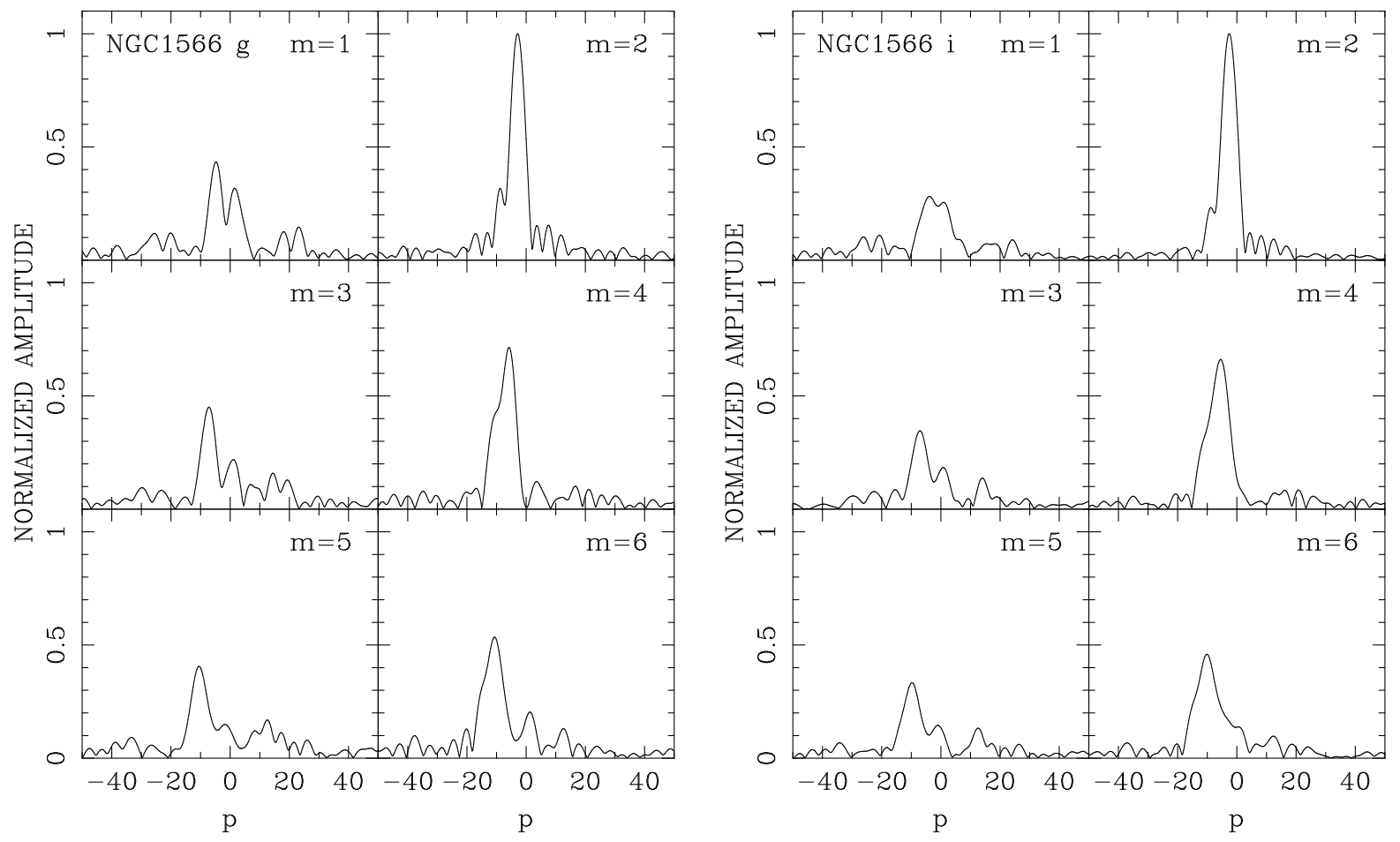

Fig. 9.- Two-dimensional Fourier spectra coefficients $A(p, m)$ of NGC 1566, obtained as described in section 2.4.2. Left, $g$ image, and right $i$ image. The $p$ variable is related to the pitch angle $\alpha$ by $p=-\frac{m}{\tan \alpha}$. 
Fig. 10. - NGC $1566 \mathrm{~g}$ band rectified image in $\log \mathrm{r}$ versus $\theta$ diagram. The horizontal line shows the CR loci at $9.4 \mathrm{kpc}$. According to EEM92. 


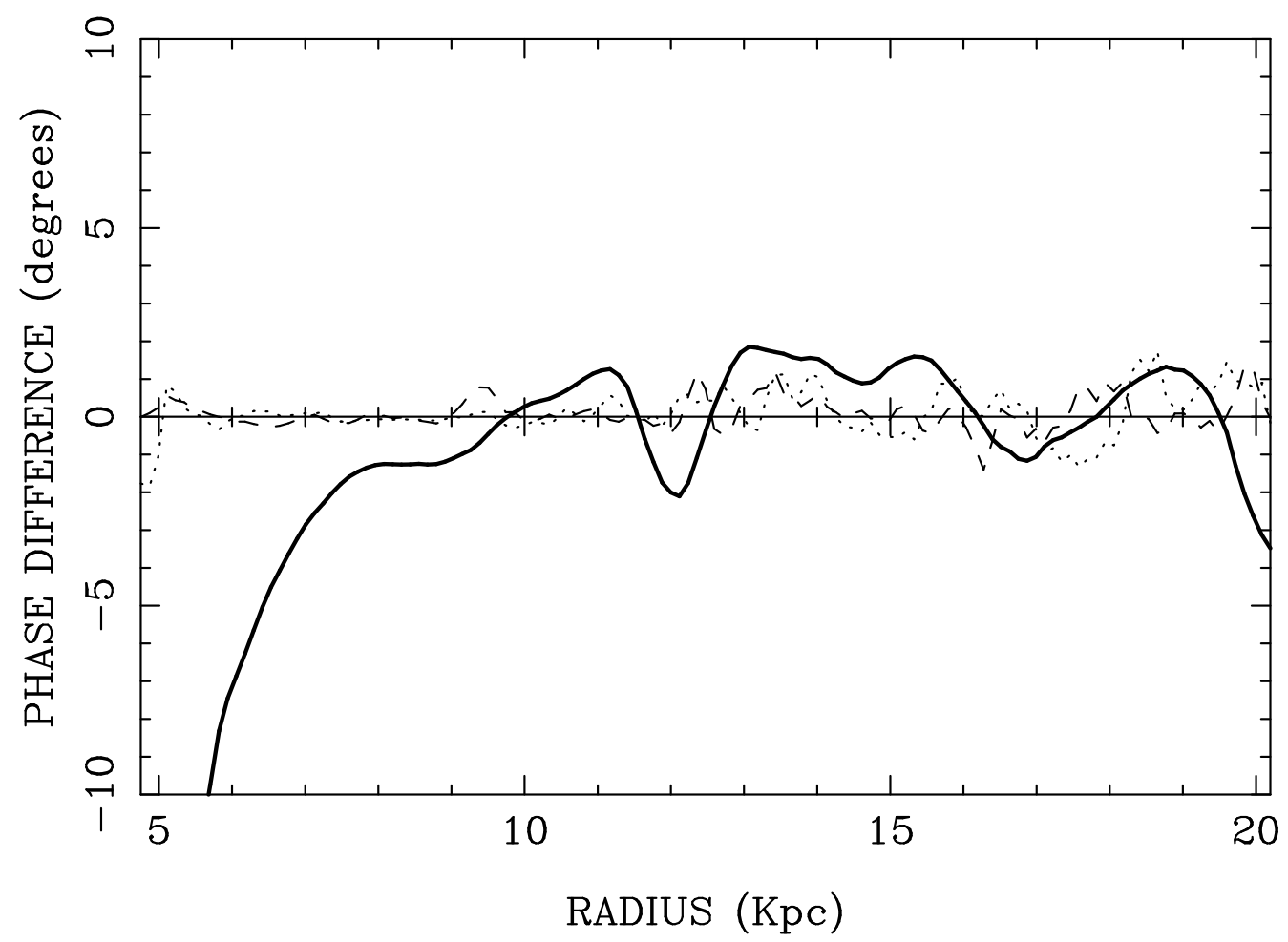

Fig. 11.- APPD $\left[\Theta_{2 g}-\Theta_{2 i}\right]$ for NGC 1566 obtained from original images (solid line). Broken line shows $g$ noise and dotted line shows $i$ noise. 


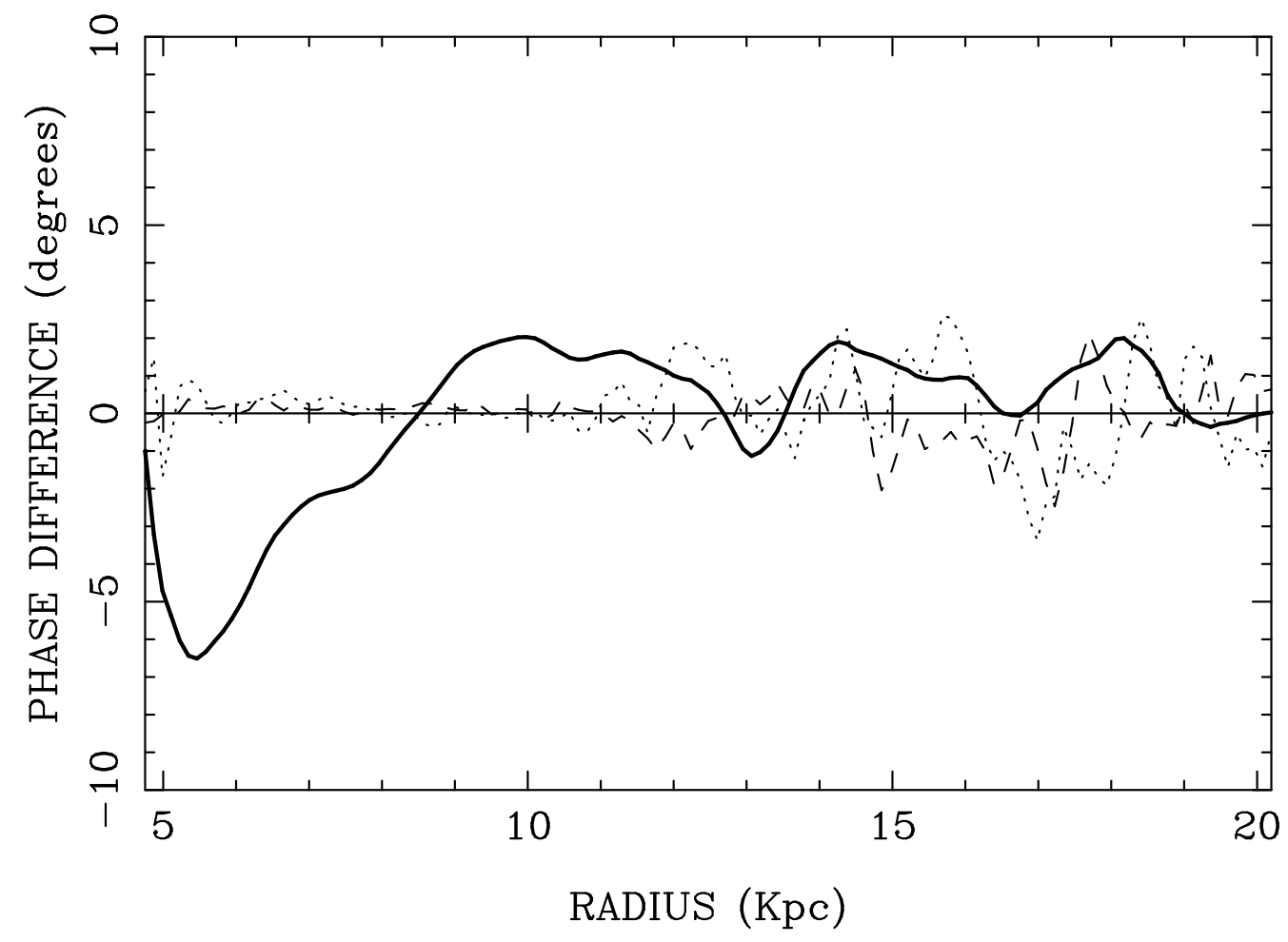

Fig. 12.- APPD $\left[\Theta_{2 g}-\Theta_{2 i}\right]$ for NGC 1566 obtained from images symmetrized according to EEM92 Method (solid line). Broken line shows the noise in $g$ color and dotted line shows that in $i$ color. See sections 2.4.1 and 2.3. 


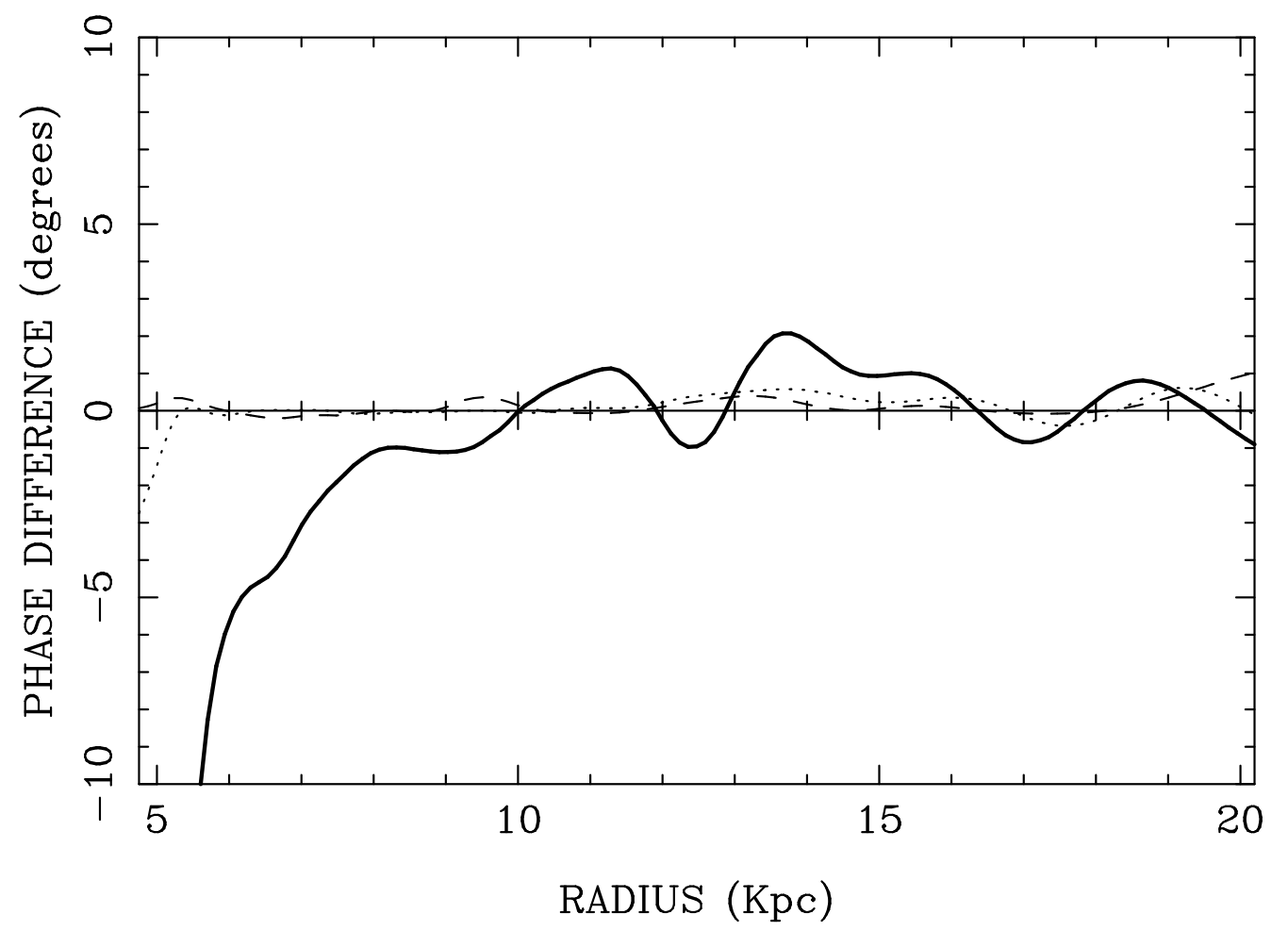

Fig. 13.- APPD $\left[\Theta_{2 g}-\Theta_{2 i}\right]$ for NGC 1566 obtained from the Fourier $\mathrm{m}=2$ modes (solid line). Broken line shows $g$ noise and dotted line shows $i$ noise. See section 2.4.2. 


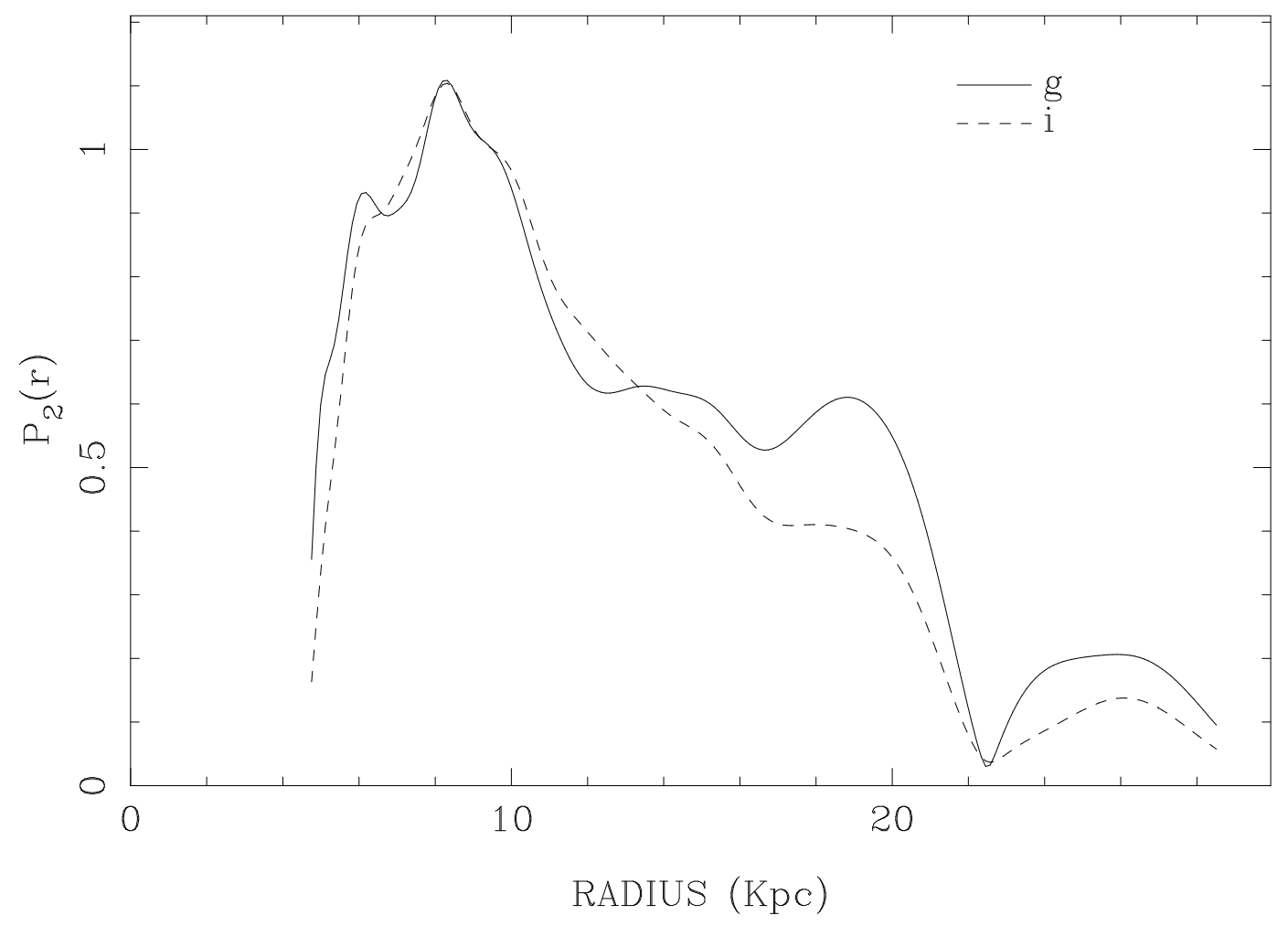

Fig. 14. - Comparison of Fourier radial density functions in blue $P_{2 g}$ and infrared $P_{2 i}$ (see section 2.4.2) for NGC 1566. 


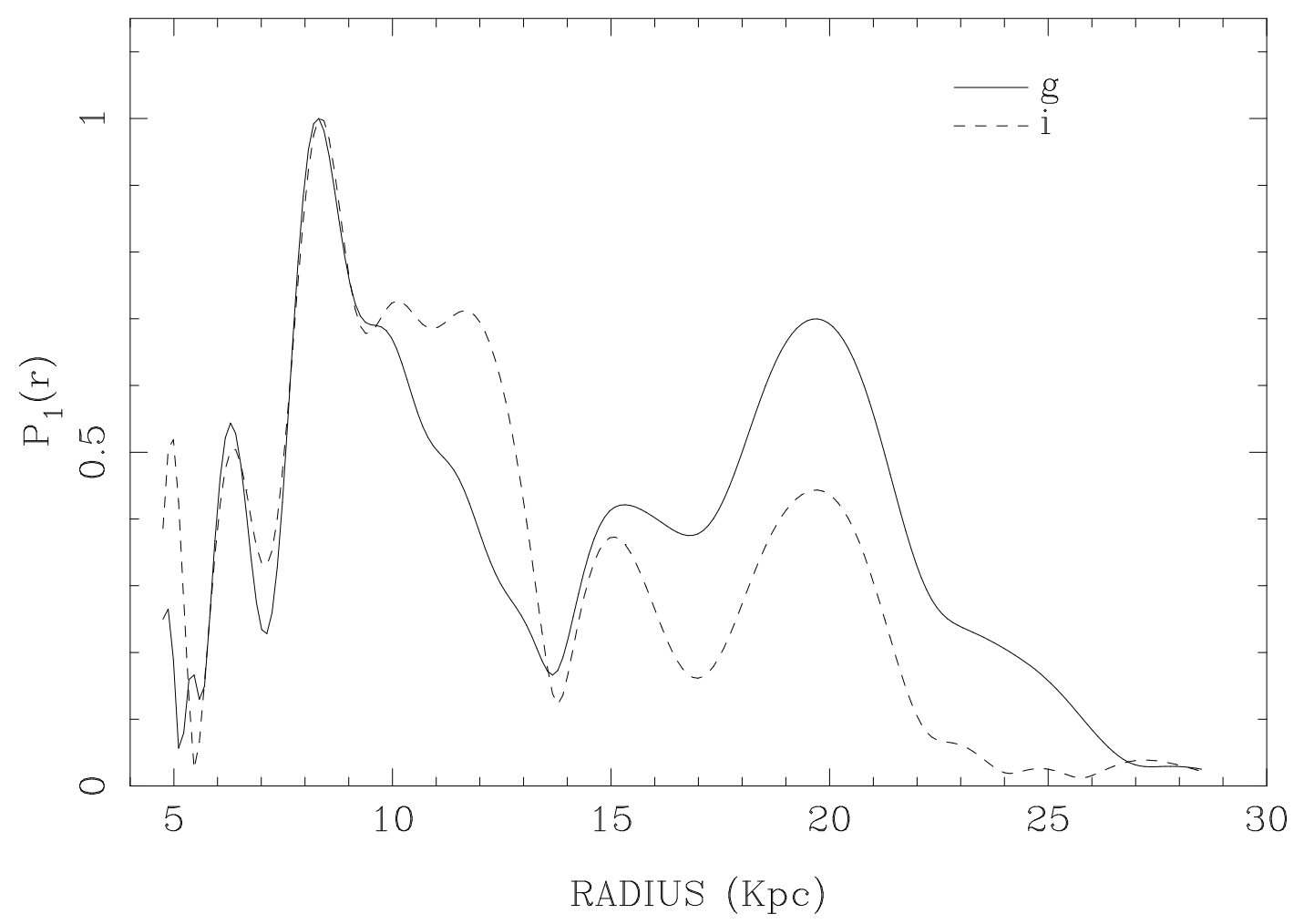

Fig. 15.- Comparison of Fourier radial density functions in blue $P_{1 g}(r)$ and infrared $P_{1 i}(r)$ (see section 2.4.2) for NGC 1566. 
(a)

(b)

Fig. 16. - NGC 2997 disk subtracted and rectified to face-on images according to procedure described in section 2.1. (a) $g$ image, (b) $i$ image. 

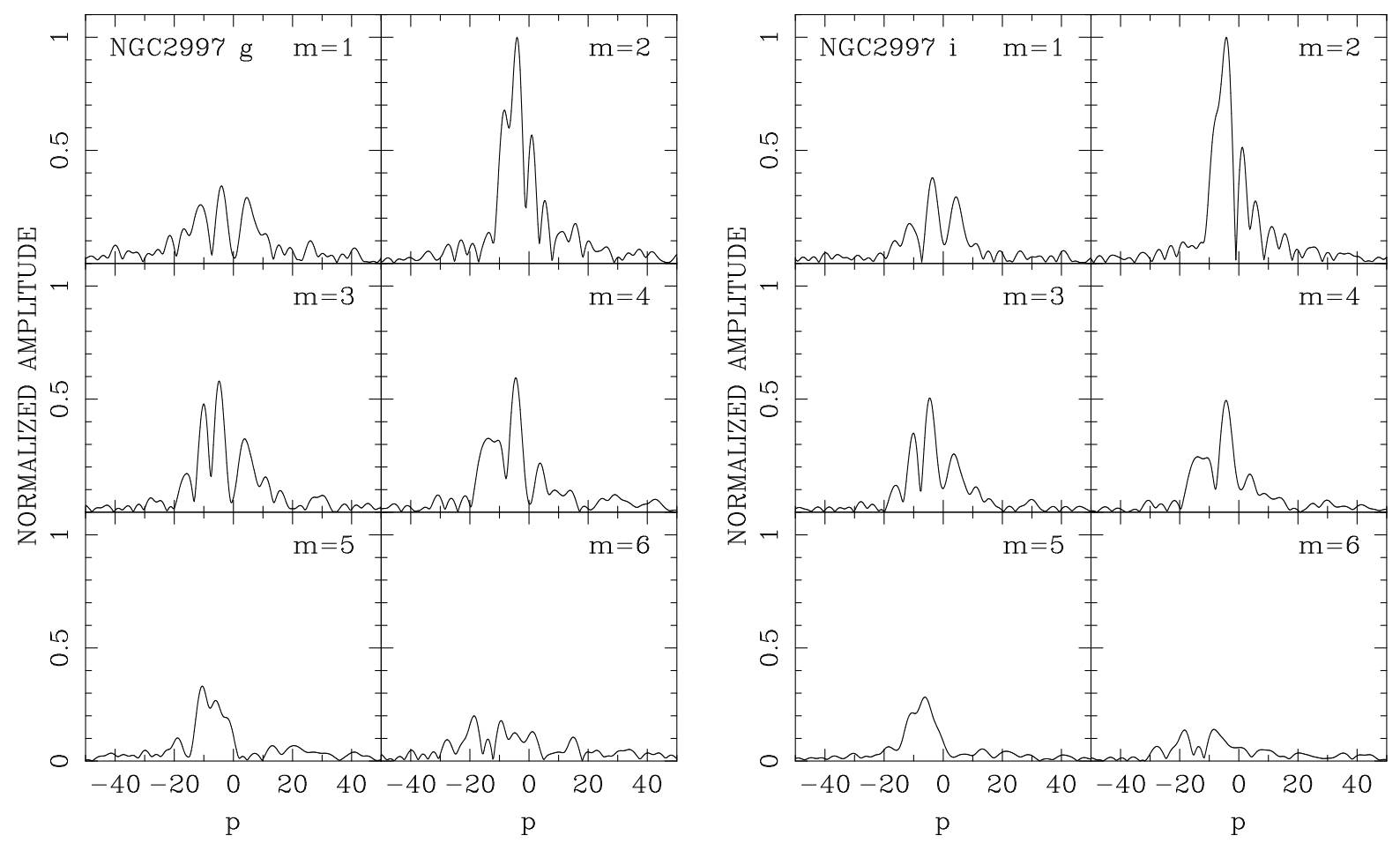

Fig. 17.- Two-dimensional Fourier spectra coefficients $A(p, m)$ of NGC 2997, obtained as described in section 2.4.2. Left, $g$ image, and right $i$ image. The $p$ variable is related to the pitch angle $\alpha$ by $p=-\frac{m}{\tan \alpha}$. 
Fig. 18. - NGC $2997 g$ band symmetrized EEM92 S2 and rectified image in log R versus $\theta$ diagram. The horizontal line shows the CR loci at $7 \mathrm{kpc}$. According to EEM92. 
Fig. 19. - NGC 2997 S2 symmetrized image according to EEM92 Method. 


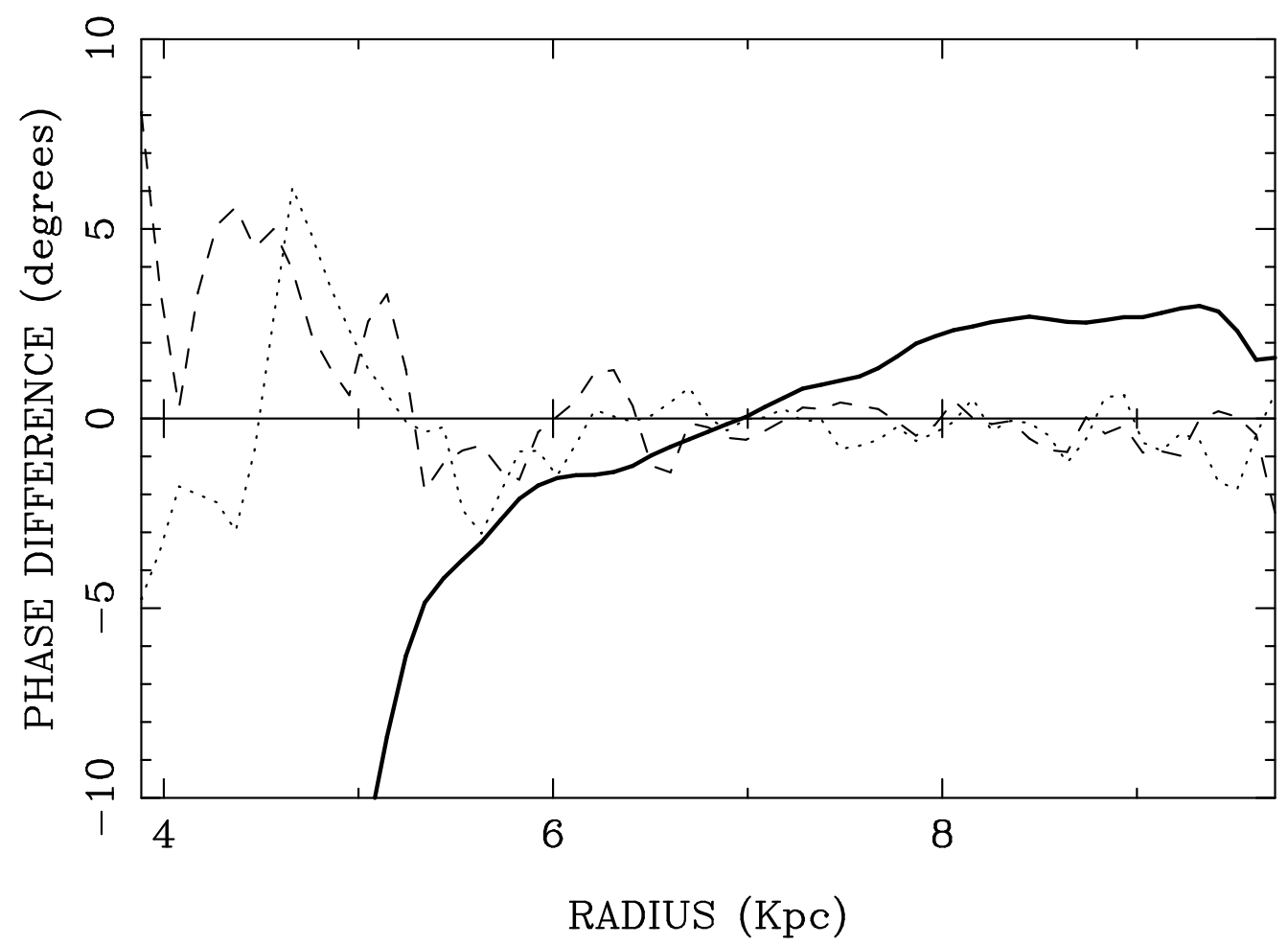

Fig. 20.- APPD $\left[\Theta_{2 g}-\Theta_{2 i}\right]$ for NGC 2997 obtained from images symmetrized according to EEM92 Method, inner arms between $4<r<11 \mathrm{kpc}$ (solid line). Broken line shows the noise in $g$ color and dotted line shows that in $i$ color. 


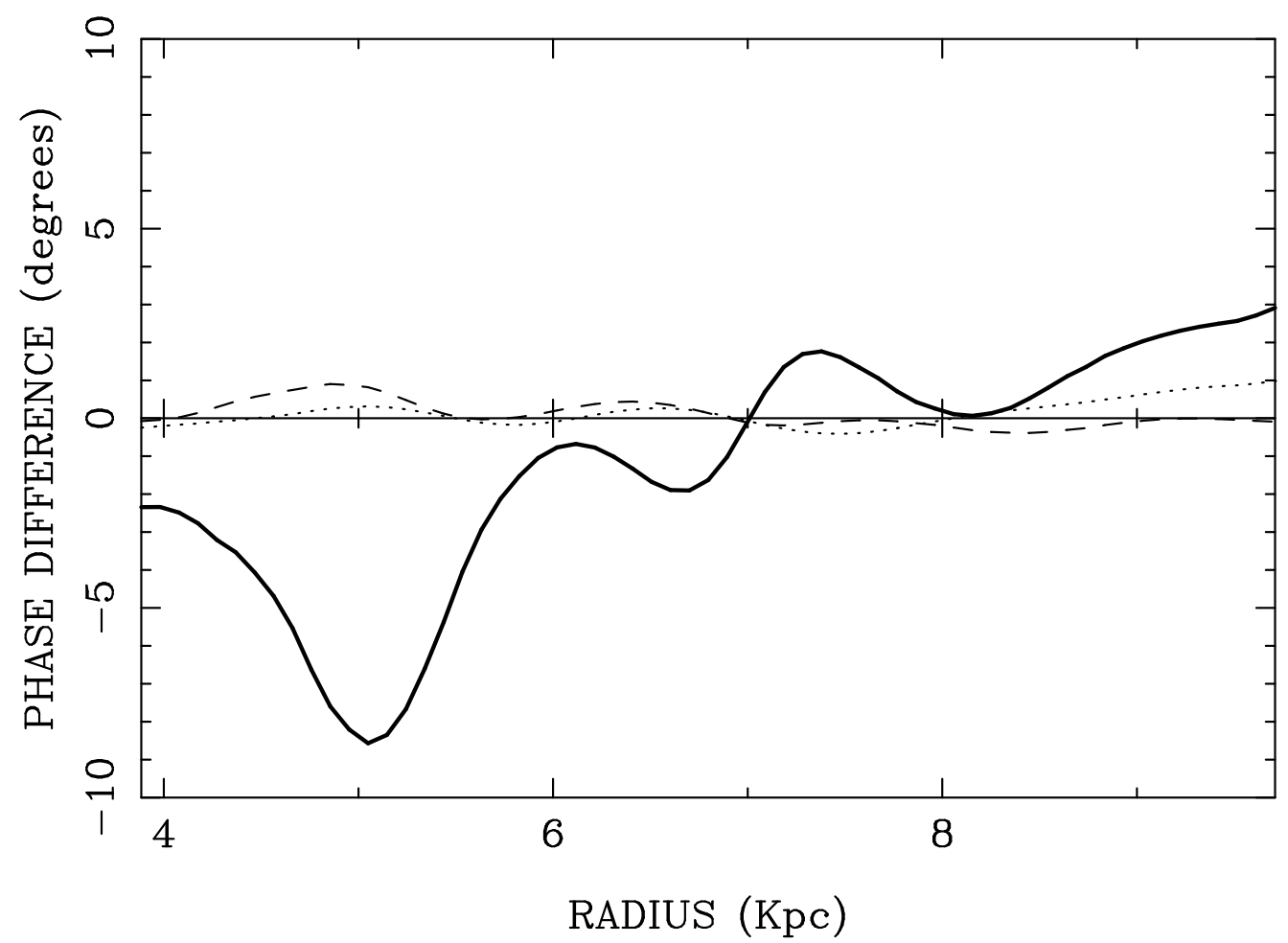

Fig. 21. - APPD $\left[\Theta_{2 g}-\Theta_{2 i}\right]$ for NGC 2997 obtained from the Fourier $\mathrm{m}=2$ modes (solid line). Broken line shows $g$ noise and dotted line shows $i$ noise. See section 2.4.2. 


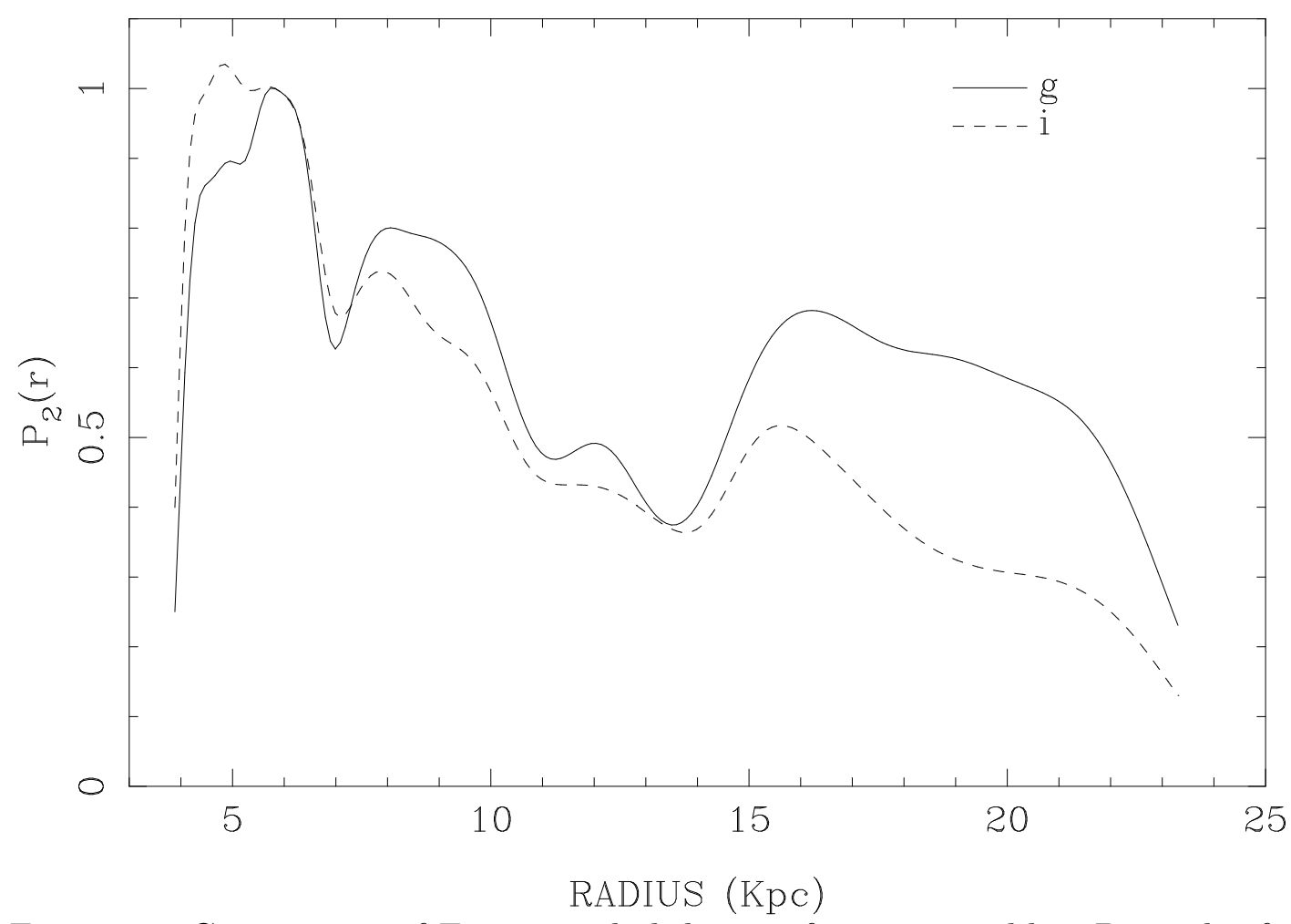

Fig. 22.- Comparison of Fourier radial density functions in blue $P_{2 g}$ and infrared $P_{2 i}$ (see section 2.4.2) for NGC 2997. 


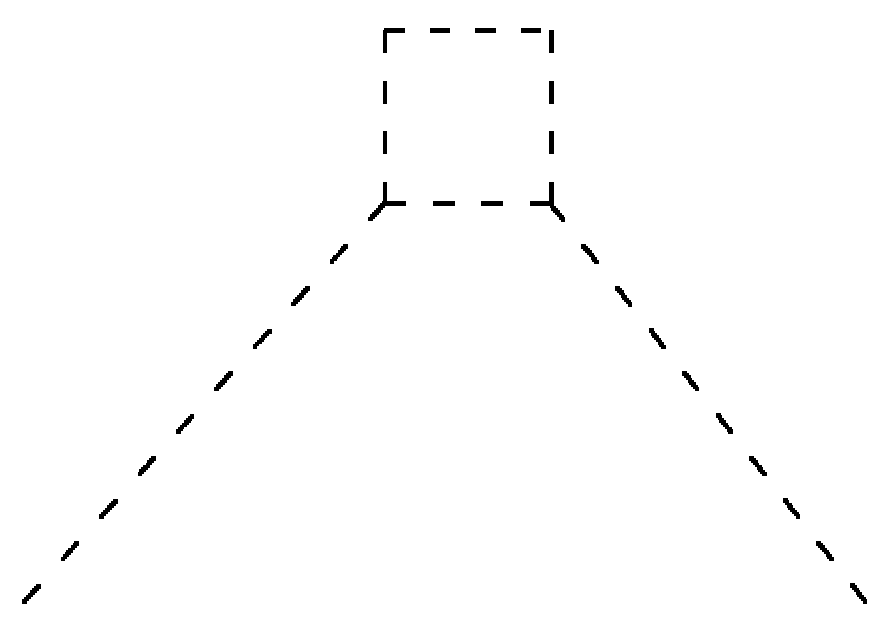

Fig. 23.- NGC 2997 S3 symmetrized image according to EEM92 Method. Curves drawn at the 3 -arm beginning points to the leading fronts of star formation. The blow-up shows details of one of the three fronts. See section 5.1 paragraph 3. 


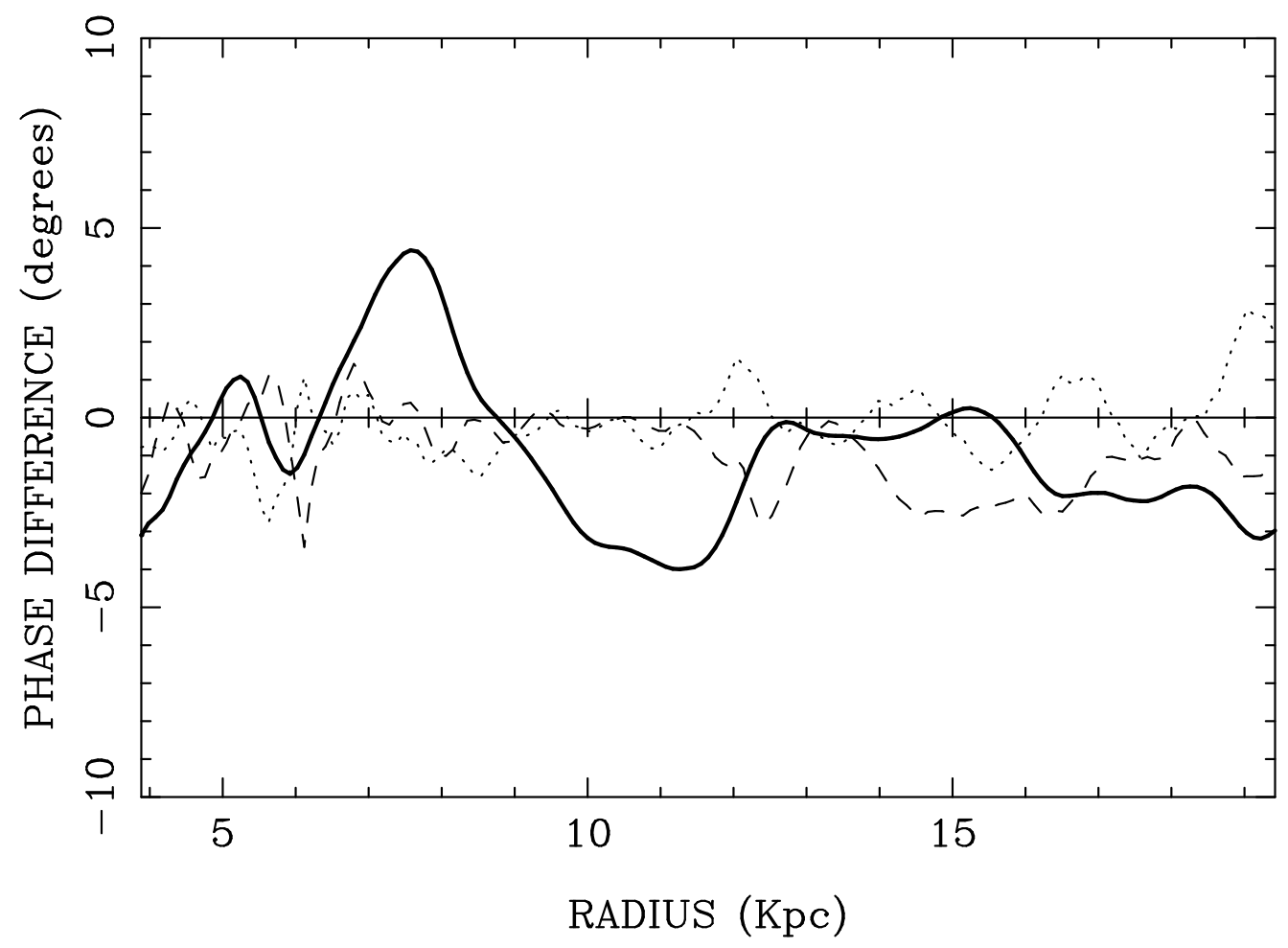

Fig. 24.- APPD $\left[\Theta_{3 g}-\Theta_{3 i}\right]$ for NGC 2997 obtained from $\mathrm{m}=3$ Fourier modes (solid line). This Fourier analysis was performed in S3 symmetrized images according to EEM92 Method. Broken line shows the noise in $g$ color and dotted line shows that in $i$ color. 
Table 1. Galaxy parameters.

\begin{tabular}{cccccccc}
\hline \hline Galaxy & Type & RA & Dec & $\mathrm{i}^{\circ}$ & $\mathrm{PA}^{\circ}$ & $\mathrm{v}(\mathrm{km} / \mathrm{s})$ & Conv $\left(\mathrm{kpc} /{ }^{\prime \prime}\right)$ \\
\hline \multirow{2}{*}{ NGC 1365 } & $\mathrm{SBb}(\mathrm{s}) \mathrm{b}$ & 03h33m36.4s & $-36 \mathrm{~d} 08 \mathrm{~m} 25 \mathrm{~s}$ & 40 & 220 & 1636 & 0.1074 \\
NGC 1566 & $\mathrm{SAB}(\mathrm{rs}) \mathrm{bc}$ & 04h20m00.6s & $-54 \mathrm{~d} 56 \mathrm{~m} 17 \mathrm{~s}$ & 37 & 60 & 1496 & 0.0967 \\
NGC 2997 & $\mathrm{SA}(\mathrm{s}) \mathrm{c}$ & 09h45m38.6s & $-31 \mathrm{~d} 11 \mathrm{~m} 25 \mathrm{~s}$ & 40 & 80 & 1087 & 0.073 \\
\hline
\end{tabular}


Table 2. Resonances mean value. $\mathrm{h}=75 \mathrm{Km} / \mathrm{s} / \mathrm{Mpc}$

\begin{tabular}{lccccc}
\hline \hline Galaxy & CR & ILR & OLR & $\mathrm{m}$ & $\Omega_{C R}(k m / s / k p c)$ \\
\hline & & & & & \\
NGC1365 $^{\mathrm{a}}$ & $12.1 \pm 0.9$ & 0.6 & 18.3 & 2 & $25.0 \pm 1.9$ \\
NGC1566 $^{\mathrm{b}}$ & $9.4 \pm 0.4$ & 3.2 & 14.6 & 2 & $12.2 \pm 0.9$ \\
& $7.1^{\mathrm{c}}$ & $\ldots$ & 14.8 & 1 & 16.6 \\
NGC2997 $^{\mathrm{d}}$ & 7.0 & 2.7 & 9.7 & 2 & 17.6 \\
& 8.7 & 6.0 & 10.9 & 3 & 12.7 \\
& & & & & \\
\hline
\end{tabular}

aThe rotation curve was taken from Persic \& Salucci (1995) and Jörsäter \& van Moorsel (1995).

b The rotation curve was taken from Persic \& Salucci (1995). ${ }^{\mathrm{c}}$ One-arm CR was estimated from Log $\mathrm{r}$ vs $\Theta$ diagram of the antisymmetric images $g$ and $i$ according to Elmegreen, Elmegreen \& Montenegro method. The $\mathrm{S} / \mathrm{N}$ is low and the value should be taken with ware.

dThe rotation curve was taken from Sperandio et al. (1995). 
This figure "fig01a.gif" is available in "gif" format from: http://arxiv.org/ps/astro-ph/0009191v1 
This figure "fig01b.gif" is available in "gif" format from: http://arxiv.org/ps/astro-ph/0009191v1 
This figure "fig03.gif" is available in "gif" format from: http://arxiv.org/ps/astro-ph/0009191v1 
This figure "fig08a.gif" is available in "gif" format from: http://arxiv.org/ps/astro-ph/0009191v1 
This figure "fig08b.gif" is available in "gif" format from: http://arxiv.org/ps/astro-ph/0009191v1 
This figure "fig10.gif" is available in "gif" format from: http://arxiv.org/ps/astro-ph/0009191v1 
This figure "fig16a.gif" is available in "gif" format from: http://arxiv.org/ps/astro-ph/0009191v1 
This figure "fig16b.gif" is available in "gif" format from: http://arxiv.org/ps/astro-ph/0009191v1 
This figure "fig18.gif" is available in "gif" format from: http://arxiv.org/ps/astro-ph/0009191v1 
This figure "fig19.gif" is available in "gif" format from: http://arxiv.org/ps/astro-ph/0009191v1 
This figure "fig23a.gif" is available in "gif" format from: http://arxiv.org/ps/astro-ph/0009191v1 
This figure "fig23b.gif" is available in "gif" format from: http://arxiv.org/ps/astro-ph/0009191v1 